\title{
Numerical analysis of the Half-Space Matching method with Robin traces on a convex polygonal scatterer
}

\author{
Anne-Sophie Bonnet-Ben Dhia ${ }^{1}$, Sonia Fliss ${ }^{1}$, and Yohanes Tjandrawidjaja ${ }^{1,2}$ \\ ${ }^{1}$ POEMS (CNRS-INRIA-ENSTA ParisTech), Palaiseau, France \\ ${ }^{2} \mathrm{CEA}$ - LIST, Saclay, France
}

We consider the 2D Helmholtz equation with a complex wavenumber in the exterior of a convex polygonal obstacle, with a Robin type boundary condition. Using the principle of the Half-Space Matching method, the problem is formulated as a system of coupled Fourier-integral equations, the unknowns being the Robin traces on the infinite straight lines supported by the edges of the polygon. We prove that this system is a Fredholm equation of the second kind, in an $L^{2}$ functional framework. The truncation of the Fourier integrals and the finite element approximation of the corresponding numerical method are also analyzed. The theoretical results are supported by various numerical experiments.

Keywords: Helmholtz equation, Fourier-integral operators, Fredholm equation, Mellin transform, error estimates

\section{Introduction}

\subsection{Motivation}

This study takes place in the general framework of the development of numerical methods for the simulation and the optimization of ultrasonic Non-Destructive Testing (NDT) experiments. NDT consists in detecting defects in an elastic structure by measuring the ultrasonic echoes produced by these defects, when they are illuminated by some incident ultrasonic wave. In particular, one needs to simulate the interaction of a given incident wave with a compactly supported defect in an infinite medium. When this medium is homogeneous and isotropic, there exist several efficient methods to solve this problem, like Perfectly Matched Layers or integral equations. However, difficulties arise in more complex configurations $[5,7,21]$. Among them, one important case which remains unsolved is the case where the infinite medium is an infinite elastic plate made of an anisotropic homogeneous material.

A new method called the Half-Space Matching (HSM) method (inspired by [13]) has been introduced recently in view of tackling this problem. As a first step, the method has been applied in [8] to the acoustic scalar problem in $\mathbb{R}^{2}$, showing that anisotropy can be taken into account easily, without any additional cost. The method mainly relies on a decomposition of the infinite domain, exterior to the obstacle, into the union of several overlapping half-spaces, where a Fourier-integral representation of the solution is available.

In this first version of the method, the unknowns of the Fourier-integral equations are the Dirichlet traces of the field on the boundaries of the different half-spaces. But with this choice, the method cannot be extended to the case of the elastic plate, where both the traces of the displacement and of the normal stress are required to derive the half-plate representations. This is a first motivation of the present paper where we consider still a scalar problem but with different types of traces, including Neumann, Dirichlet, and Robin traces. 
The content of the present paper is the following. We first derive the HSM formulation for general types of traces. Then we prove the well-posedness of the continuous problem by adapting the arguments used in [8]. More importantly, the main contribution of this paper is the numerical analysis of the discretized formulation which is not straightforward and has never been addressed in previous works.

The model problem that we consider is presented in the next subsection. The corresponding HSM formulation is the object of Section 2. Section 3 is devoted to the theoretical analysis of the formulation: we use Fredholm theory, the main tools being the Mellin transform [12,17] and Hilbert-Schmidt operators $[19$, p.210]. The discretization aspects are detailed in Section 4 and error estimates are derived for an appropriate Fourier discretization. Some numerical results are finally presented in Section 5.

\subsection{The model problem}

The problem that we consider is the $2 \mathrm{D}$ Helmholtz equation in the exterior of a compact convex polygonal obstacle $\mathcal{O}$, with a boundary condition of Robin type. More precisely, the problem takes the following form where $\omega, \alpha$ and $\beta$ are some complex constants whose characteristics are specified below, $\nu$ denotes the outgoing normal to $\mathcal{O}$ and the data is a given function $g$ defined on the boundary of the obstacle $\partial \mathcal{O}$ :

$$
\mid \begin{array}{ll}
\Delta p+\omega^{2} p=0 & \text { in } \Omega=\mathbb{R}^{2} \backslash \mathcal{O} \\
\alpha p+\beta \frac{\partial p}{\partial \nu}=g & \text { on } \partial \mathcal{O} .
\end{array}
$$

In the sequel, we will use the following assumptions:

$$
\operatorname{Im} \omega>0, \beta \neq 0, \operatorname{Im}\left(\frac{\alpha}{\beta \omega}\right) \geq 0, \text { and } g \in L^{2}(\partial \mathcal{O}),
$$

which lead to several results as follows.

1. Since $\operatorname{Im} \omega>0$ (which can be justified in a dissipative medium), we will look for a solution $p$ which belongs to $H^{1}(\Omega)$. More precisely, $p$ is exponentially decaying at infinity and satisfies

$$
\forall \varepsilon<\operatorname{Im}(\omega), \quad(x, y) \mapsto p(x, y) e^{\varepsilon \sqrt{x^{2}+y^{2}}} \in H^{1}(\Omega) .
$$

However, we emphasize that the numerical method also works in the non-dissipative case, that is when $\omega \in \mathbb{R}^{+}$. In this latter case, $p$ is chosen as the outgoing solution of (1) (defined as the unique solution satisfying the Sommerfeld condition) .

2. As $\beta \neq 0$, the problem (1) admits the following variational formulation:

$$
\begin{array}{r}
\text { Find } p \in H^{1}(\Omega) \text { such that for all } q \in H^{1}(\Omega) \\
\int_{\Omega} \nabla p \cdot \overline{\nabla q}-\omega^{2} \int_{\Omega} p \bar{q}-\frac{\alpha}{\beta} \int_{\partial \mathcal{O}} p \bar{q}=\frac{-1}{\beta} \int_{\partial \mathcal{O}} g \bar{q} .
\end{array}
$$

Using the fact that for $p \in H^{1}(\Omega)$ :

$$
\begin{aligned}
& \operatorname{Im}\left(\frac{-1}{\omega}\left(\int_{\Omega}|\nabla p|^{2}-\omega^{2}|p|^{2}-\frac{\alpha}{\beta} \int_{\partial \mathcal{O}}|p|^{2}\right)\right)= \\
& \frac{\operatorname{Im}(\omega)}{|\omega|^{2}} \int_{\Omega}|\nabla p|^{2}+\operatorname{Im}(\omega) \int_{\Omega}|p|^{2}+\operatorname{Im}\left(\frac{\alpha}{\beta \omega}\right) \int_{\partial \mathcal{O}}|p|^{2},
\end{aligned}
$$

one deduces, due to the assumption that $\operatorname{Im}\left(\frac{\alpha}{\beta \omega}\right) \geq 0$, that the bilinear form is coercive. Then the problem is well-posed by Lax-Milgram theorem. 
Remark 1. For the data $g$ on the boundary, we make the assumption $g \in L^{2}(\partial \mathcal{O})$, which is convenient for our approach, and which differs from the natural one $\left(g \in H^{-\frac{1}{2}}(\partial \mathcal{O})\right)$ that would be used in a variational approach. In particular, since $g \in L^{2}(\partial \mathcal{O})$, we know from classical regularity results [15] that $p \in H^{3 / 2}(\Omega)$.

Remark 2 (The Dirichlet case). Taking $\beta=0$ and $\alpha \neq 0$ in (1), one simply recovers a Dirichlet boundary condition (a case which has been already treated in [8]). In that case, the natural hypothesis in a variational approach would be $g \in H^{\frac{1}{2}}(\partial \mathcal{O})$. We point out that our approach allows to consider more general Dirichlet data which are only in $L^{2}(\partial \mathcal{O})$. As a consequence, the solution may not be in $H^{1}$ up to the boundary (see [1] for a similar problem). Note that the numerical analysis performed in Section 4 is also valid in the Dirichlet case, which is illustrated numerically in Section 5.3.1.

\section{The Half-Space Matching formulation}

The Half-Space Matching method consists in coupling several analytical representations of the solution in half-planes surrounding the obstacle.

\subsection{Geometry and notations}

Let us consider a convex polygon $\mathcal{O}$ with $n$ edges $\Sigma_{\mathcal{O}}^{j}, j=0, \ldots, n-1$. For convenience, we introduce $\mathbb{Z} / n \mathbb{Z}$ the ring of integers modulo $n$. For $j \in \mathbb{Z} / n \mathbb{Z}$, the angle between $\Sigma_{\mathcal{O}}^{j}$ and $\Sigma_{\mathcal{O}}^{j+1}$ is denoted as $\theta^{j, j+1}$ or equivalently $\theta^{j+1, j}$. Because of the convexity, one has

$$
0<\theta^{j, j+1}<\pi
$$

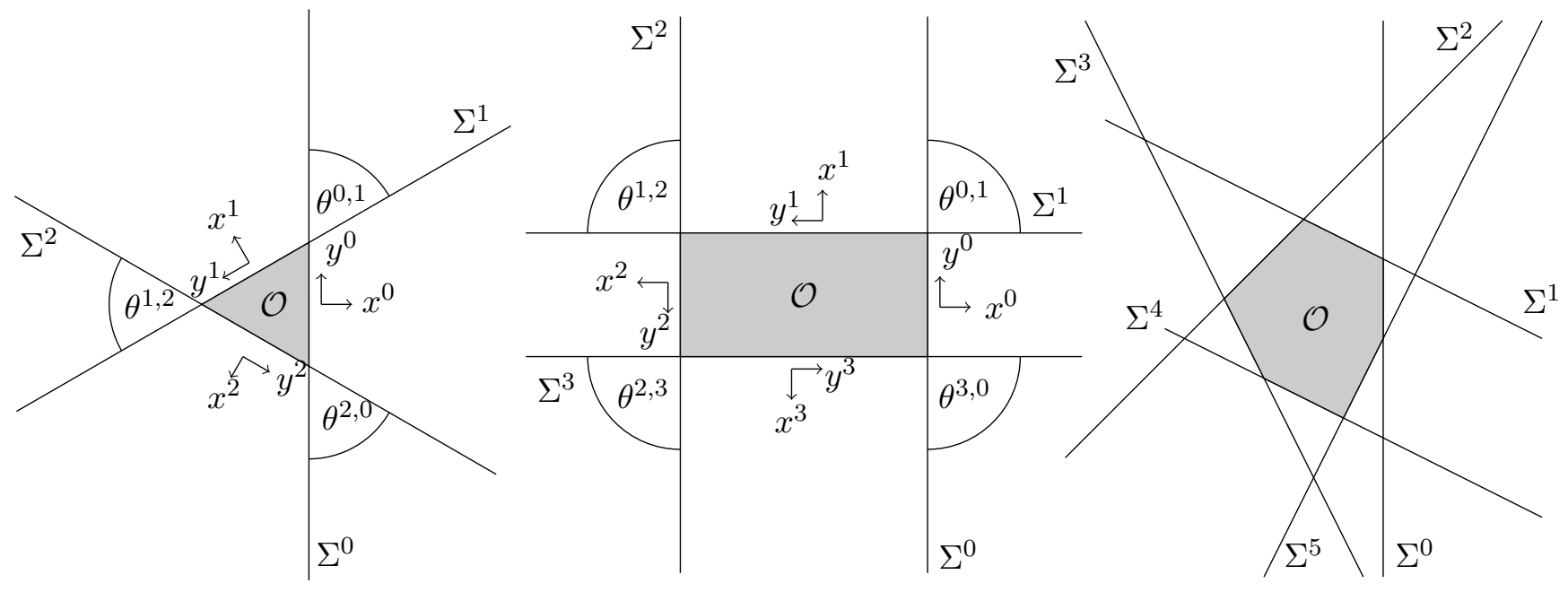

Figure 1: Examples of polygons $\mathcal{O}$ for $n=3,4$ and 6 and associated notations.

To define the half-spaces, we introduce several local coordinate systems $\left(x^{j}, y^{j}\right)$. The origin of all of them is the centroid $O$ of the polygon $\mathcal{O}$. We choose the reference Cartesian coordinate system $\left(O, \mathbf{e}_{x}^{0}, \mathbf{e}_{y}^{0}\right)$ such that $\mathbf{e}_{x}^{0}$ is orthogonal to $\Sigma_{\mathcal{O}}^{0}$ and oriented to the exterior of the polygon, while the axis $\mathbf{e}_{y}^{0}$ is $\pi / 2$ counter clockwise to $\mathbf{e}_{x}^{0}$. The other local coordinate systems $\left(O, \mathbf{e}_{x}^{j}, \mathbf{e}_{y}^{j}\right)$ are defined recursively as follows:

$$
\forall j \in \mathbb{Z} / n \mathbb{Z}, \quad \mid \begin{aligned}
& \mathbf{e}_{x}^{j+1}=-\cos \theta^{j, j+1} \mathbf{e}_{x}^{j}+\sin \theta^{j, j+1} \mathbf{e}_{y}^{j}, \\
& \mathbf{e}_{y}^{j+1}=-\sin \theta^{j, j+1} \mathbf{e}_{x}^{j}-\cos \theta^{j, j+1} \mathbf{e}_{y}^{j} .
\end{aligned}
$$


If we define $l^{j}$ as the distance of the centroid of the polygon to the edge $\Sigma_{\mathcal{O}}^{j}$, each half-plane $\Omega^{j}$ is defined in the local coordinate system $\left(O, \mathbf{e}_{x}^{j}, \mathbf{e}_{y}^{j}\right)$ as

$$
\forall j \in \mathbb{Z} / n \mathbb{Z}, \quad \Omega^{j}=\left\{x^{j} \geq l^{j}\right\} \times\left\{y^{j} \in \mathbb{R}\right\},
$$

and its boundary denoted by $\Sigma^{j}$ is given by

$$
\Sigma^{j}=\left\{x^{j}=l^{j}\right\} \times\left\{y^{j} \in \mathbb{R}\right\} .
$$

\subsection{Half-space problems}

The $j^{\text {th }}$ half-space problem is defined as follows: given $\psi \in L^{2}\left(\Sigma^{j}\right), P^{j}(\psi)$ is the unique solution in $H^{1}\left(\Omega^{j}\right)$ of

$$
\mid \begin{aligned}
& \Delta P^{j}+\omega^{2} P^{j}=0, \quad \text { in } \Omega^{j} \\
& \alpha P^{j}+\beta \frac{\partial P^{j}}{\partial x^{j}}=\psi \quad \text { on } \Sigma^{j}
\end{aligned}
$$

This problem is well-posed under assumptions (2) for the same reasons than the ones detailed in Section 1.2. Remark again that in the usual framework, we would take $\psi^{j} \in H^{-1 / 2}\left(\Sigma^{j}\right)$, but here we take $\psi^{j} \in L^{2}\left(\Sigma^{j}\right)$. Applying the Fourier transform in $y^{j}$ defined as

$$
\forall \psi^{j} \in L^{2}\left(\Sigma^{j}\right), \quad \hat{\psi}^{j}(\xi)=\frac{1}{\sqrt{2 \pi}} \int_{\mathbb{R}} \psi^{j}\left(y^{j}\right) e^{-\imath \xi y^{j}} \mathrm{~d} y^{j},
$$

we obtain the following ordinary differential equation in $x^{j}$, parametrized by the Fourier variable $\xi$ :

$$
\mid \begin{array}{r}
\frac{\partial^{2} \hat{P}^{j}}{\left(\partial x^{j}\right)^{2}}+\left(\omega^{2}-\xi^{2}\right) \hat{P}^{j}=0, \quad x^{j}>l^{j} \\
\alpha \hat{P}^{j}+\beta \frac{\partial \hat{P}^{j}}{\partial x^{j}}=\hat{\psi}^{j}, \quad x^{j}=l^{j}
\end{array}
$$

whose unique $L^{2}$ solution is given by

$$
\hat{P}^{j}\left(x^{j}, \xi\right)=A(\xi) e^{\imath \sqrt{\omega^{2}-\xi^{2}}\left(x^{j}-l^{j}\right)},
$$

where $\operatorname{Im} \sqrt{\omega^{2}-\xi^{2}}>0$ and

$$
\left(\alpha+\imath \beta \sqrt{\omega^{2}-\xi^{2}}\right) A(\xi)=\hat{\psi}(\xi) .
$$

One can check that, thanks to assumptions (2), the quantity $\alpha+\imath \beta \sqrt{\omega^{2}-\xi^{2}}$ never vanishes for $\xi \in \mathbb{R}$. Finally, by taking the inverse Fourier transform, the solution $P^{j}(\psi)$ of $(7)$ is given by

$$
P^{j}\left(x^{j}, y^{j}\right)=\frac{1}{\sqrt{2 \pi}} \int_{\mathbb{R}} \frac{\hat{\psi}(\xi)}{\alpha+\imath \beta \sqrt{\omega^{2}-\xi^{2}}} e^{\imath \sqrt{\omega^{2}-\xi^{2}}\left(x^{j}-l^{j}\right)} e^{i \xi y^{j}} \mathrm{~d} \xi .
$$

\subsection{Half-space matching integral equations}

For the solution $p$ of problem (1), let us define the Robin traces

$$
\forall j \in \mathbb{Z} / n \mathbb{Z}, \quad \varphi^{j}:=\left.\left(\alpha p+\beta \frac{\partial p}{\partial x^{j}}\right)\right|_{\Sigma^{j}} .
$$

Note that $\varphi^{j} \in L^{2}\left(\Sigma^{j}\right)$ since $p \in H^{3 / 2}(\Omega)$. Our objective is to derive integral equations linking the $\varphi^{j}$ by using half-space representations of Section 2.2 and the fact that the half-spaces $\Omega^{j}$ overlap. First, the restriction of $p$ in $\Omega^{j}$ is the solution of (7) for $\psi=\varphi^{j}$. By uniqueness,

$$
\left.p\right|_{\Omega^{j}}=P^{j}\left(\varphi^{j}\right) .
$$


Then the quantity

$$
\left.\left(\alpha p+\beta \frac{\partial p}{\partial x^{j \pm 1}}\right)\right|_{\Sigma^{j \pm 1} \cap \Omega^{j}}
$$

is equal both to

$$
\left.\left.\varphi^{j \pm 1}\right|_{\Sigma^{j \pm 1} \cap \Omega^{j}} \quad \text { ( by definition of } \varphi^{j \pm 1}\right)
$$

and to

$$
\alpha P^{j}\left(\varphi^{j}\right)+\left.\beta \frac{\partial P^{j}\left(\varphi^{j}\right)}{\partial x^{j \pm 1}}\right|_{\Sigma^{j \pm 1} \cap \Omega^{j}} \quad(\text { by }(14)) .
$$

This provides the compatibility relations

$$
\varphi^{j \pm 1}=\alpha P^{j}\left(\varphi^{j}\right)+\beta \frac{\partial P^{j}\left(\varphi^{j}\right)}{\partial x^{j \pm 1}} \text { on } \Sigma^{j \pm 1} \cap \Omega^{j}, \quad \forall j \in \mathbb{Z} / n \mathbb{Z} .
$$

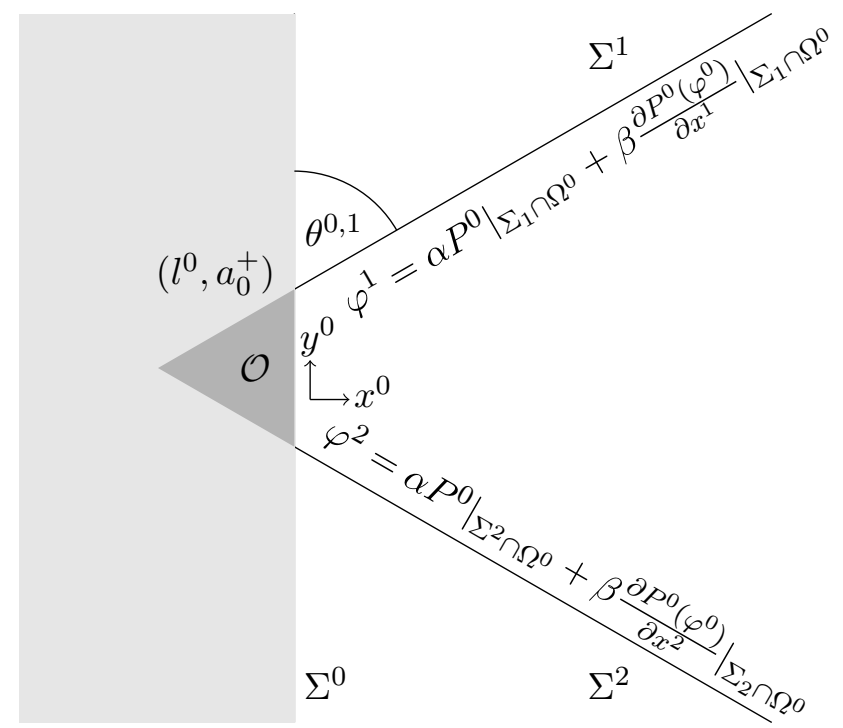

Figure 2: Robin traces on $\Sigma^{1} \cap \Omega^{0}$ and $\Sigma^{2} \cap \Omega^{0}$.

Remark 3. - Such compatibility relations have been firstly introduced in [6,13] for Dirichlet traces in the case of periodic media.

- Here we have used the overlap of two consecutive half-spaces $\Omega^{j}$ and $\Omega^{j \pm 1}$. This will be sufficient for our formulation, even for polygons with more than four edges where non-consecutive halfspaces may overlap (see Figure 1 on the right).

This leads to introducing the following integral operator

$$
\begin{aligned}
D^{j, j \pm 1}: L^{2}\left(\Sigma^{j}\right) & \rightarrow L^{2}\left(\Sigma^{j \pm 1} \cap \Omega^{j}\right) \\
\psi & \rightarrow \alpha P^{j}(\psi)+\left.\beta \frac{\partial P^{j}(\psi)}{\partial x^{j \pm 1}}\right|_{\Sigma^{j \pm 1} \cap \Omega^{j}},
\end{aligned}
$$

which can be expressed, using (12), as a kernel operator acting on the Fourier transform

$$
\left[D^{j, j \pm 1} \psi\right](r)=\frac{1}{\sqrt{2 \pi}} \int_{\mathbb{R}} k^{j, j \pm 1}(r, \xi) \hat{\psi}(\xi) \mathrm{d} \xi, \quad r \geq 0
$$

with the following kernel

$$
k^{j, j \pm 1}(r, \xi)=\frac{\alpha+\beta\left(-\cos \left(\theta^{j, j \pm 1}\right) \imath \sqrt{\omega^{2}-\xi^{2}}+\sin \left(\theta^{j, j \pm 1}\right) \imath \xi\right)}{\alpha+\imath \beta \sqrt{\omega^{2}-\xi^{2}}} e^{\imath \sqrt{\omega^{2}-\xi^{2}} r \sin \left(\theta^{j, j \pm 1}\right)} e^{\imath \xi\left(a_{j}^{ \pm}+r \cos \left(\theta^{j, j \pm 1}\right)\right)} .
$$


Here, $a_{j}^{ \pm}$denotes the ordinate of the intersection point of $\Sigma^{j}$ and $\Sigma^{j \pm 1}$ in $\left(x^{j}, y^{j}\right)$ local coordinates and $r$ is the radial variable of the polar coordinates centered at this intersection point. If $\theta^{j, j+1}=\pi / 2$ (which holds for instance for all $j$ when $\mathcal{O}$ is a rectangle), the previous operator has the simpler form

$$
\left[D^{j, j \pm 1} \psi\right](r)=\frac{1}{\sqrt{2 \pi}} \int_{\mathbb{R}} \frac{\alpha+\imath \beta \xi}{\alpha+\imath \beta \sqrt{\omega^{2}-\xi^{2}}} e^{\imath \sqrt{\omega^{2}-\xi^{2}}} r e^{\imath \xi a_{j}^{ \pm}} \hat{\psi}(\xi) \mathrm{d} \xi .
$$

It is not so difficult to see that the operator $D^{j, j \pm 1}$ is continuous from $L^{2}\left(\Sigma^{j}\right)$ to $L^{2}\left(\Omega^{j} \cap \Sigma^{j \pm 1}\right)$. Indeed, if $\psi \in L^{2}\left(\Sigma^{j}\right)$, we can show that $P^{j}(\psi)$, the solution of the half-space problem $(7)$ in $\Omega^{j}$, is in $H^{3 / 2}\left(\Omega^{j}\right)$. It suffices then to use the continuity of the trace operators. Let us remark that it is less obvious when using directly the expression (19-20) of $D^{j, j \pm 1}$, but this will be a by-product of the next section.

Summing up, we have finally the following system of coupled equations satisfied by the $\varphi^{j}$ 's:

$$
\varphi^{j}=\left\{\begin{array}{ll}
D^{j-1, j} \varphi^{j-1} & \text { on } \Sigma^{j} \cap \Omega^{j-1} \\
g & \text { on } \Sigma_{\mathcal{O}}^{j} \\
D^{j+1, j} \varphi^{j+1} & \text { on } \Sigma^{j} \cap \Omega^{j+1},
\end{array} \quad \forall j \in \mathbb{Z} / n \mathbb{Z}\right.
$$

where we have used the boundary condition satisfied by $p$ on $\partial \mathcal{O}$. The system of equations (22) can be written in a matricial form as

$$
(\mathbb{I}-\mathbb{D}) \Phi=G
$$

where

$$
\Phi \in V:=\left\{\left(\varphi^{0}, \varphi^{1}, \ldots, \varphi^{n-1}\right) \in \prod_{j=0}^{n-1} L^{2}\left(\Sigma^{j}\right)\right\},
$$

$\mathbb{I}$ corresponds to the identity operator and $\mathbb{D}$ is given by

$$
\mathbb{D}:=\left[\begin{array}{cccccc}
0 & D^{1,0} & 0 & \ldots & 0 & D^{n-1,0} \\
D^{0,1} & 0 & D^{2,1} & \ldots & 0 & 0 \\
0 & D^{1,2} & 0 & \ldots & 0 & 0 \\
\vdots & \vdots & \vdots & \ddots & \vdots & \vdots \\
0 & 0 & 0 & \ldots & 0 & D^{n-1, n-2} \\
D^{0, n-1} & 0 & 0 & \ldots & D^{n-2, n-1} & 0
\end{array}\right]
$$

where for all $j \in \mathbb{Z} / n \mathbb{Z}$ we have identified a function of $L^{2}\left(\Sigma^{j} \cap \Omega^{j \pm 1}\right)$ to a function of $L^{2}\left(\Sigma^{j}\right)$ by extending it by 0 . Remark then that for all $\Phi$ in $V, \mathbb{D} \Phi$ is in $\tilde{V}$ where

$$
\tilde{V}:=\left\{\tilde{\Phi}=\left(\tilde{\varphi}^{0}, \tilde{\varphi}^{1}, \ldots, \tilde{\varphi}^{n-1}\right) \in V, \tilde{\varphi}^{j}=0 \text { on } \Sigma_{\mathcal{O}}^{j} \quad \forall j \in \mathbb{Z} / n \mathbb{Z}\right\} .
$$

Remark 4. If we want to make the extension by 0 explicit, we have to replace in $\mathbb{D}, D^{j, j \pm 1}$ by $E^{j, j \pm 1} D^{j, j \pm 1}$ where

$$
\begin{aligned}
E^{j, j \pm 1}: L^{2}\left(\Sigma^{j \pm 1} \cap \Omega^{j}\right) & \rightarrow L^{2}\left(\Sigma^{j \pm 1}\right) \\
\psi & \mapsto E^{j, j \pm 1} \psi
\end{aligned}
$$

with

$$
E^{j, j \pm 1} \psi=\left\{\begin{array}{l}
\psi \text { on } \Sigma^{j \pm 1} \cap \Omega^{j} \\
0 \text { on } \Sigma^{j \pm 1} \backslash\left(\Sigma^{j \pm 1} \cap \Omega^{j}\right) .
\end{array}\right.
$$

All the properties of $D^{j, j \pm 1}$ also hold trivially for $E^{j, j \pm 1} D^{j, j \pm 1}$. In order to enhance readability, we have chosen to drop these extension operators.

Lemma 5 (Equivalence). Let $g \in L^{2}(\partial \mathcal{O})$. If $p \in H^{1}(\Omega)$ is solution of $(1)$ then $\Phi=\left(\varphi^{0}, \varphi^{1}, \ldots, \varphi^{n-1}\right)$ where $\varphi^{j}$ is defined by (13) belongs to $V$ and is a solution of (23).

Conversely, if $\Phi \in V$ is a solution of (23), then $p$ satisfying (14) for all $j \in \mathbb{Z} / n \mathbb{Z}$ is a function defined "unequivocally" in $\Omega$. Moreover, $p \in H^{1}(\Omega)$ and is solution of (1). 
Proof. The first assertion is true by construction. Conversely, suppose that $\Phi=\left(\varphi^{0}, \ldots, \varphi^{n-1}\right) \in V$ is solution of (23). This implies that the $\varphi^{j}$ 's satisfy the system of coupled equations (22). Now, let us introduce $P^{j}\left(\varphi^{j}\right) \in H^{1}\left(\Omega^{j}\right)$ for all $j \in \mathbb{Z} / n \mathbb{Z}$, the solution of the half-space problem (7) with $\psi=\varphi^{j}$. By definition:

$$
\varphi^{j}=\alpha P^{j}\left(\varphi^{j}\right)+\left.\beta \frac{\partial P^{j}\left(\varphi^{j}\right)}{\partial x^{j}}\right|_{\Sigma^{j}} .
$$

Because the $\varphi^{j}$ 's satisfy the first set of equations of (22), we have by definition of $D^{j \pm 1, j}$ that

$$
\varphi^{j}=\alpha P^{j \pm 1}\left(\varphi^{j \pm 1}\right)+\beta \frac{\partial P^{j \pm 1}\left(\varphi^{j \pm 1}\right)}{\partial x^{j}} \text { on } \Sigma^{j} \cap \Omega^{j \pm 1}, \quad \forall j \in \mathbb{Z} / n \mathbb{Z} .
$$

From (28) and (29), we have that

$$
\alpha P^{j}\left(\varphi^{j}\right)+\left.\beta \frac{\partial P^{j}\left(\varphi^{j}\right)}{\partial x^{j}}\right|_{\Sigma^{j} \cap \Omega^{j \pm 1}}=\alpha P^{j \pm 1}\left(\varphi^{j \pm 1}\right)+\left.\beta \frac{\partial P^{j \pm 1}\left(\varphi^{j \pm 1}\right)}{\partial x^{j}}\right|_{\Sigma^{j} \cap \Omega^{j \pm 1}} .
$$

In particular, the previous relations for $j=0$ and $\pm=+$ and for $j=1$ and $\pm=-$ yield to

$$
\alpha P^{0}\left(\varphi^{0}\right)+\left.\beta \frac{\partial P^{0}\left(\varphi^{0}\right)}{\partial x^{0}}\right|_{\Sigma^{0} \cap \Omega^{1}}=\alpha P^{1}\left(\varphi^{1}\right)+\left.\beta \frac{\partial P^{1}\left(\varphi^{1}\right)}{\partial x^{0}}\right|_{\Sigma^{0} \cap \Omega^{1}}
$$

and

Let

$$
\alpha P^{1}\left(\varphi^{1}\right)+\left.\beta \frac{\partial P^{1}\left(\varphi^{1}\right)}{\partial x^{1}}\right|_{\Sigma^{1} \cap \Omega^{0}}=\alpha P^{0}\left(\varphi^{0}\right)+\left.\beta \frac{\partial P^{0}\left(\varphi^{0}\right)}{\partial x^{1}}\right|_{\Sigma^{1} \cap \Omega^{0}}
$$

$$
Q=P^{0}\left(\varphi^{0}\right)-P^{1}\left(\varphi^{1}\right) \quad \text { in } \Omega^{0} \cap \Omega^{1} .
$$

Because $P^{0}\left(\varphi^{0}\right)$ and $P^{1}\left(\varphi^{1}\right)$ satisfy the same Helmholtz equation and because of the previous relations, $Q$ satisfies the problem

$$
\mid \begin{array}{ll}
\Delta Q+\omega^{2} Q=0 & \text { in } \Omega^{0} \cap \Omega^{1} \\
\alpha Q+\beta \frac{\partial Q}{\partial \nu}=0 & \text { on } \partial\left(\Omega^{0} \cap \Omega^{1}\right),
\end{array}
$$

where $\nu$ is the interior normal to $\Omega^{0} \cap \Omega^{1}$. This problem is well-posed under assumptions (2) for the same reasons as the ones detailed in Section 1.2. So $Q=0$ in $\Omega^{0} \cap \Omega^{1}$ which means that $P^{0}\left(\varphi^{0}\right)$ and $P^{1}\left(\varphi^{1}\right)$ coincide in the overlapping zone $\Omega^{0} \cap \Omega^{1}$.

Similar arguments enable us to show that for all $j \in \mathbb{Z} / n \mathbb{Z}, P^{j}\left(\varphi^{j}\right)$ and $P^{j+1}\left(\varphi^{j+1}\right)$ coincide in the overlapping zone $\Omega^{j} \cap \Omega^{j+1}$. We can then define unequivocally a function $p$ by

$$
\forall j \in \mathbb{Z} / n \mathbb{Z},\left.\quad p\right|_{\Omega^{j}}=P^{j}\left(\varphi^{j}\right) .
$$

Because the half-space solutions coincide two by two in the overlapping zones, the function $p$ is in $H^{1}(\Omega)$ and is solution of the Helmholtz equation in $\Omega$. Moreover, by definition

$$
\forall j \in \mathbb{Z} / n \mathbb{Z},\left.\quad\left(\alpha p+\beta \frac{\partial p}{\partial x^{j}}\right)\right|_{\Sigma_{\mathcal{O}}^{j}}=\left.\left(\alpha P^{j}\left(\varphi^{j}\right)+\beta \frac{\partial P^{j}\left(\varphi^{j}\right)}{\partial x^{j}}\right)\right|_{\Sigma_{\mathcal{O}}^{j}}=\left.\varphi^{j}\right|_{\Sigma_{\mathcal{O}}^{j}}=g,
$$

where the last equality is obtained by using the second set of equations of (22). Hence, the function $p$ is then solution of (1).

Finally,

$$
G:=\left(g^{0}, g^{1}, \ldots, g^{n-1}\right) \in V \quad \text { where } g^{j}= \begin{cases}g & \text { on } \Sigma_{\mathcal{O}}^{j} \\ 0 & \text { otherwise }\end{cases}
$$

Writing

$$
\Phi=G+\tilde{\Phi}
$$

where $\tilde{\Phi}$ is in $\tilde{V}$, we obtain an equivalent system

$$
(\mathbb{I}-\mathbb{D}) \tilde{\Phi}=\mathbb{D} G .
$$

This system constitutes the Half-Space Matching formulation which will be analyzed in Section 3 . 


\section{Analysis of the continuous formulation}

In this section, we consider the general problem

$$
\text { Find } \tilde{\Phi} \in \tilde{V}, \quad(\mathbb{I}-\mathbb{D}) \tilde{\Phi}=F,
$$

where $\tilde{V}$ is defined in $(26), \mathbb{D}$ is defined in $(25)$, and $F \in \tilde{V}$. Denoting $\mathcal{L}(A)$ as the set of bounded linear operators of a vector space $A$, we show in this section the following main results.

Theorem 6. The operator $(\mathbb{I}-\mathbb{D}) \in \mathcal{L}(\tilde{V})$ is the sum of a coercive operator and a compact one. Moreover, Problem (33) is well-posed.

A naive idea would be that $\mathbb{D} \in \mathcal{L}(\tilde{V})$ is compact, but it is not. However, it can be decomposed as the sum of an operator of norm strictly less than 1 and a compact operator. This decomposition is linked to a similar decomposition of the operators $D^{j, j \pm 1}$. Inspired by the proofs for the Dirichlet case shown in [8], we prove the properties of the operators for the Robin case in Section 3.1 and finally show the theorem in Section 3.2.

\subsection{Properties of the operators $D^{j, j \pm 1}$}

Let us concentrate first on the operator $D^{0,1}$ and similar properties will be given, without proof, for all the operators $D^{j, j \pm 1}$ at the end of this section. To simplify the notation, we denote in this section, $D^{0,1}=D, x^{0}=x, y^{0}=y$. We will identify, when necessary, $\Sigma^{0}$ to $\mathbb{R}$, its upper part $\Sigma^{0} \cap \Omega^{1}$ to $\left(a_{0}^{+},+\infty\right)$, its lower part $\Sigma^{0} \cap \Omega^{n-1}$ to $\left(-\infty, a_{0}^{-}\right)$and finally $\Sigma^{1} \cap \Omega^{0}$ to $\mathbb{R}^{+}$. Let us also introduce for any open interval $I$ included in $J$, an open interval of $\mathbb{R}$, the restriction operator $\chi_{I}$

$$
\begin{aligned}
\chi_{I}: L^{2}(J) & \rightarrow L^{2}(J) \\
\varphi & \mapsto \mid \begin{array}{l}
\chi_{I} \varphi=\varphi \text { on } I \\
\chi_{I} \varphi=0 \text { on } J \backslash I
\end{array}
\end{aligned}
$$

In the sequel, we are going to decompose the operator $D$ progressively in order to isolate a compact part and a part for which we get the norm explicitly.

First, from the definition (17), we can decompose simply $D$ as

$$
D=D_{D}+D_{N}
$$

where

$$
\begin{aligned}
D_{D}: L^{2}\left(\Sigma^{0}\right) & \rightarrow L^{2}\left(\Sigma^{1} \cap \Omega^{0}\right) \\
\psi & \left.\mapsto \alpha P^{0}(\psi)\right|_{\Sigma^{1} \cap \Omega^{0}}
\end{aligned} \text { and } \begin{aligned}
D_{N}: L^{2}\left(\Sigma^{0}\right) & \rightarrow L^{2}\left(\Sigma^{1} \cap \Omega^{0}\right) \\
\psi & \left.\mapsto \beta \frac{\partial P^{0}(\psi)}{\partial x^{1}}\right|_{\Sigma^{1} \cap \Omega^{0}} .
\end{aligned}
$$

Lemma 7. The operator $D_{D}: L^{2}\left(\Sigma^{0}\right) \rightarrow L^{2}\left(\Sigma^{1} \cap \Omega^{0}\right)$ is compact.

Proof. By definition of $D_{D}$, we have

$$
\forall \psi \in L^{2}\left(\Sigma^{0}\right), \quad D_{D} \psi(r)=\int_{\mathbb{R}} k_{D}(\xi, r) \hat{\psi}(\xi) \mathrm{d} \xi
$$

with

$$
k_{D}(\xi, r)=\frac{\alpha}{\alpha+\imath \beta \sqrt{\omega^{2}-\xi^{2}}} e^{\imath \sqrt{\omega^{2}-\xi^{2}} r \sin \theta^{0,1}} e^{\imath \xi\left(a_{0}^{+}+r \cos \theta^{0,1}\right)} .
$$

Using Fubini's theorem, we obtain

$$
\begin{aligned}
\left\|k_{D}(\xi, r)\right\|_{L^{2}\left(\mathbb{R} \times \mathbb{R}^{+}\right)}^{2} & =\int_{\mathbb{R}} \int_{\mathbb{R}^{+}} \frac{|\alpha|^{2}}{\left|\alpha+\imath \beta \sqrt{\omega^{2}-\xi^{2}}\right|^{2}} e^{-2 \operatorname{Im}\left(\sqrt{\omega^{2}-\xi^{2}}\right) r \sin \theta^{0,1}} \mathrm{~d} r \mathrm{~d} \xi \\
& =\int_{\mathbb{R}} \frac{|\alpha|^{2}}{2 \operatorname{Im}\left(\sqrt{\omega^{2}-\xi^{2}}\right) \sin \theta\left|\alpha+\imath \beta \sqrt{\omega^{2}-\xi^{2}}\right|^{2}} \mathrm{~d} \xi \\
& <+\infty
\end{aligned}
$$


This proves that $D_{D}$ is the composition of the Fourier operator $\psi \mapsto \hat{\psi}$ and of a Hilbert-Schmidt operator. The lemma follows.

Let us focus now on $D_{N}$. For all $\psi, D_{N} \psi$ is, up to the parameter $\beta$, the normal trace on $\Sigma^{1} \cap \Omega^{0}$ of the half-space solution $P^{0}(\psi)$ in $\Omega^{0}$ with a Robin data $\psi$ on the boundary $\Sigma^{0}$. Because the half-line $\Sigma^{1} \cap \Omega^{0}$ touches $\Sigma^{0}$, the operator $D_{N}$ is not compact. The lack of compactness is precisely due to the intersection point. So let us isolate the intersection point by decomposing $D_{N}$ thanks to restriction operators:

$$
D_{N}=\chi_{(0, b)} D_{N}+\chi_{(b,+\infty)} D_{N}, \quad \text { with } b>0 .
$$

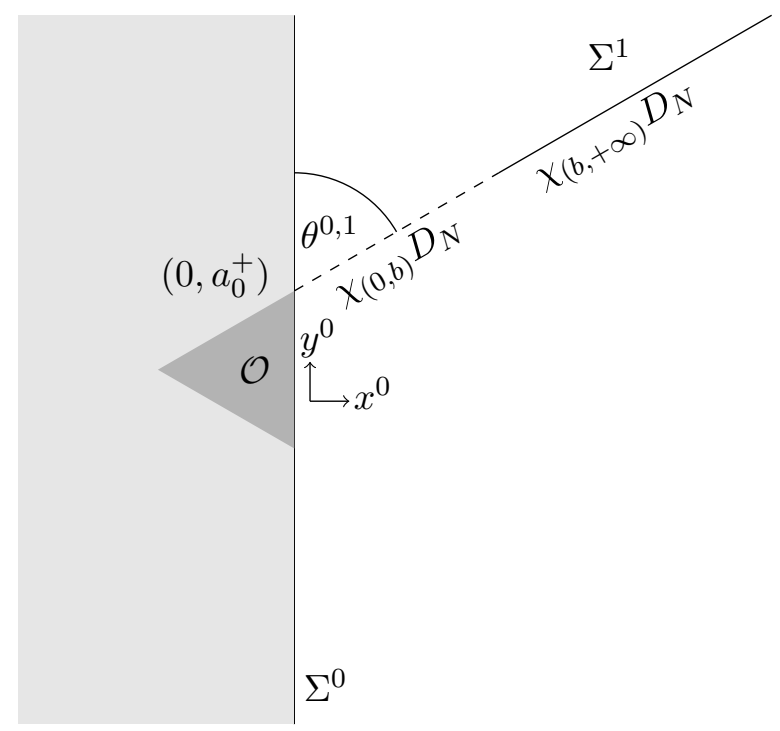

Figure 3: Decomposition of the operator $D_{N}$ into $\chi_{(0, b)} D_{N}$ and $\chi_{(b,+\infty)} D_{N}$ with a regular triangle as the obstacle.

Lemma 8. For any $b>0$, the operator $\chi_{(b,+\infty)} D_{N}: L^{2}\left(\Sigma^{0}\right) \rightarrow L^{2}\left(\Omega^{0} \cap \Sigma^{1}\right)$ is compact.

Proof. By definition of $D_{N}$, we have

$$
\forall \psi \in L^{2}\left(\Sigma^{0}\right), \quad D_{N} \psi(r)=\int_{\mathbb{R}} k_{N}(\xi, r) \hat{\psi}(\xi) \mathrm{d} \xi
$$

with

$$
k_{N}(\xi, r)=\frac{\beta\left(-\imath \sqrt{\omega^{2}-\xi^{2}} \cos \theta^{0,1}+\imath \xi \sin \theta^{0,1}\right)}{\alpha+\imath \beta \sqrt{\omega^{2}-\xi^{2}}} e^{\imath \sqrt{\omega^{2}-\xi^{2}} r \sin \theta^{0,1}} e^{\imath \xi\left(a_{0}^{+}+r \cos \theta^{0,1}\right)} .
$$

Again by Fubini's theorem, we get for $b>0$

$$
\begin{aligned}
\left\|k_{N}(\xi, r)\right\|_{L^{2}(\mathbb{R} \times(b,+\infty))}^{2} & =\int_{\mathbb{R}} \int_{b}^{+\infty} \frac{|\beta|^{2}\left|-\imath \sqrt{\omega^{2}-\xi^{2}} \cos \theta^{0,1}+\imath \xi \sin \theta^{0,1}\right|^{2}}{\left|\alpha+\imath \beta \sqrt{\omega^{2}-\xi^{2}}\right|^{2}} e^{-2 \operatorname{Im}\left(\sqrt{\omega^{2}-\xi^{2}}\right) r \sin \theta^{0,1}} \mathrm{~d} r \mathrm{~d} \xi \\
& =\int_{\mathbb{R}} \frac{|\beta|^{2}\left|-\imath \sqrt{\omega^{2}-\xi^{2}} \cos \theta^{0,1}+\imath \xi \sin \theta^{0,1}\right|^{2}}{2 \operatorname{Im}\left(\sqrt{\omega^{2}-\xi^{2}}\right) \sin \theta^{0,1}\left|\alpha+\imath \beta \sqrt{\omega^{2}-\xi^{2}}\right|^{2}} e^{-2 \operatorname{Im}\left(\sqrt{\omega^{2}-\xi^{2}}\right) b \sin \theta^{0,1} \mathrm{~d} \xi} \\
& <+\infty .
\end{aligned}
$$

We conclude as in the proof of Lemma 7. 
As you can notice, this proof requires that $b>0$. To analyse the non compact part $\chi_{(0, b)} D_{N}$, inspired by the Dirichlet case [8] and more generally by the singularity theory [17], we decompose finally $\chi_{(0, b)} D_{N}$ as

$$
\chi_{(0, b)} D_{N}=\chi_{(0, b)} L_{N}+\chi_{(0, b)}\left(D_{N}-L_{N}\right)
$$

where $L_{N}$ is obtained by taking $\omega=0$ in the expression (37) of $k_{N}(\xi, r)$

$$
L_{N} \psi(r)=\frac{1}{\sqrt{2 \pi}} \int_{\mathbb{R}} \hat{\psi}(\xi)\left(-\cos \theta^{0,1}-\imath \operatorname{sgn}(\xi) \sin \theta^{0,1}\right) e^{-|\xi| r \sin \theta^{0,1}} e^{\imath \xi\left(a_{0}^{+}+r \cos \theta^{0,1}\right)} \mathrm{d} \xi, \quad r>0 .
$$

The operator $L_{N}$ is similar to $D_{N}$, but it is associated with the Laplace operator. Indeed, it can also be defined as

$$
\begin{aligned}
L_{N}: L^{2}\left(\Sigma^{0}\right) & \rightarrow L^{2}\left(\Sigma^{1} \cap \Omega^{0}\right), \\
L_{N} \psi & :=\left.\beta \frac{\partial}{\partial x^{1}} v(\psi)\right|_{\Sigma^{1} \cap \Omega^{0}},
\end{aligned}
$$

where, for all $\psi \in L^{2}\left(\Sigma^{0}\right), v(\psi)$ is the solution (at least in the distributional sense) to

$$
\mid \begin{aligned}
& -\Delta v=0 \quad \text { in } \Omega^{0} \\
& \beta \frac{\partial v}{\partial x}=\psi
\end{aligned}
$$

We refer to the appendix for the precise definition of the appropriate functional framework for this problem.

Lemma 9. The operator $\chi_{(0, b)}\left(D_{N}-L_{N}\right)$ is compact.

Proof. It is a kernel operator whose kernel is given by

$$
k(\xi, r)=\left(c_{1}(\xi) e^{\imath \sqrt{\omega^{2}-\xi^{2}} r \sin \theta^{0,1}}-c_{2}(\xi) e^{-|\xi| r \sin \theta^{0,1}}\right) e^{\imath \xi\left(a^{+}+r \cos \theta^{0,1}\right)}
$$

where from (37) and (38), we have that

$$
c_{1}, c_{2}, 1 / c_{2} \in L^{\infty}(\mathbb{R}), \quad \text { and } \quad\left|\frac{c_{1}(\xi)}{c_{2}(\xi)}\right| \rightarrow 1 \text { when } \xi \rightarrow+\infty
$$

Consequently

$$
|k(\xi, r)|=\left|c_{2}(\xi)\right| e^{-|\xi| r \sin \theta^{0,1}}\left|\frac{c_{1}(\xi)}{c_{2}(\xi)} e^{-q(\xi) r \sin \theta^{0,1}}-1\right|
$$

where

$$
q(\xi)=\sqrt{\xi^{2}-\omega^{2}}-|\xi|=\frac{-\omega^{2}}{\sqrt{\xi^{2}-\omega^{2}}+|\xi|}=\mathcal{O}\left(\frac{1}{\xi}\right) .
$$

We deduce that

$$
|k(\xi, r)| \leq C|q(\xi)| e^{-|\xi| r \sin \theta^{0,1}}
$$

which enables us to conclude as in the proof of Lemma 7.

Finally, let us focus on the properties of $L_{N}$ which are summarized in this fundamental lemma.

Lemma 10. The operator $L_{N}$ is continuous from $L^{2}\left(\Sigma^{0}\right)$ to $L^{2}\left(\Sigma^{1} \cap \Omega^{0}\right)$ and its norm is bounded by 1. Moreover, we have

- $\exists C \in(0,1), \quad \forall \varphi \in L^{2}\left(\Sigma^{0}\right), \quad\left\|L_{N} \chi_{\left(a_{0}^{+},+\infty\right)} \varphi\right\| \leq C\left\|\chi_{\left(a_{0}^{+},+\infty\right)} \varphi\right\| ;$

- $L_{N} \chi_{\left(-\infty, a_{0}^{-}\right)}$is a compact operator. 
We give the proof which is quite technical in Appendix A. As we will see in the proof of Theorem 6, it is not sufficient to know that the norm of $L_{N}$ is bounded by 1 . This is the second part of the lemma which will enable us to conclude that $\mathbb{I}-\mathbb{D}$ is a sum of a coercive operator and a compact one. As indicated in the appendix, the constant $C$ is linked to the angle $\theta^{0,1}$ between $\Sigma^{0}$ and $\Sigma^{1} \cap \Omega^{0}$ :

$$
C=\cos \left(\theta^{0,1} / 2\right) \text {. }
$$

When $\theta^{0,1}$ tends to 0 , this constant tends to 1 .

Gathering all the results of this section, we can show the following properties of $D$.

Proposition 11. The operator $D$ is such that $D-L$ is a compact operator from $L^{2}\left(\Sigma^{0}\right)$ to $L^{2}\left(\Sigma^{1} \cap \Omega^{0}\right)$ where $L$ is a continuous operator from $L^{2}\left(\Sigma^{0}\right)$ to $L^{2}\left(\Sigma^{1} \cap \Omega^{0}\right)$ which satisfies

- $\exists C \in(0,1), \quad \forall \varphi \in L^{2}\left(\Sigma^{0}\right), \quad\left\|L \chi_{\left(a_{0}^{+},+\infty\right)} \varphi\right\| \leq C\left\|\chi_{\left(a_{0}^{+},+\infty\right)} \varphi\right\|$;

- $L \chi_{\left(-\infty, a_{0}^{-}\right)}$is a compact operator.

Proof. The operator $L$ is nothing else but $\chi_{(0, b)} L_{N}$. Indeed, using all the operators introduced in this section, we write

$$
D-\chi_{(0, b)} L_{N}=\chi_{(0, b)}\left(D_{N}-L_{N}\right)+\chi_{(b,+\infty)} D_{N}+D_{D} .
$$

From Lemmas 7, 8 and 9, we have that the operator $D-\chi_{(0, b)} L_{N}$ is compact. As $L_{N}$ satisfies Lemma 10, the operator $\chi_{(0, b)} L_{N}$ inherits similar properties.

Finally, we have obviously similar results for all the operators $D^{j, j \pm 1}$ for $j \in \mathbb{Z} / n \mathbb{Z}$. Again, we will identify, when necessary, $\Sigma^{j}$ to $\mathbb{R}$, its upper part $\Sigma^{j} \cap \Omega^{j+1}$ to $\left(a_{j}^{+},+\infty\right)$, its lower part $\Sigma^{j} \cap \Omega^{j-1}$ to $\left(-\infty, a_{j}^{-}\right)$. Finally, in order to give a short statement of the following theorem, we use the notation

$$
\forall j, \quad\left(a_{j}^{-},-\infty\right)=\left(-\infty, a_{j}^{-}\right) .
$$

Theorem 12. The operator $D^{j, j \pm 1}$ is such that $D^{j, j \pm 1}-L^{j, j \pm 1}$ is a compact operator from $L^{2}\left(\Sigma^{j}\right)$ to $L^{2}\left(\Sigma^{j \pm 1} \cap \Omega^{j}\right)$ where $L^{j, j \pm 1}$ is a continuous operator from $L^{2}\left(\Sigma^{j}\right)$ to $L^{2}\left(\Sigma^{j \pm 1} \cap \Omega^{j}\right)$ which satisfies

- $\exists C^{j, j \pm 1} \in(0,1), \forall \varphi \in L^{2}\left(\Sigma^{j}\right), \quad\left\|L^{j, j \pm 1} \chi_{\left(a_{j}^{ \pm}, \pm \infty\right)} \varphi\right\| \leq C^{j, j \pm 1}\left\|\chi_{\left(a_{j}^{ \pm}, \pm \infty\right)} \varphi\right\|$;

- $L^{j, j \pm 1} \chi_{\left(a_{j}^{\mp}, \mp \infty\right)}$ is a compact operator from $L^{2}\left(\Sigma^{j}\right)$ to $L^{2}\left(\Sigma^{j \pm 1} \cap \Omega^{j}\right)$.

Remark 13. The constant $C^{j, j \pm 1}$ is linked to the angle $\theta^{j, j \pm 1}$ between $\Sigma^{j}$ and $\Sigma^{j \pm 1} \cap \Omega^{j}$. More precisely, we can show, as in Appendix A, that

$$
C^{j, j \pm 1}=\cos \left(\theta^{j, j \pm 1} / 2\right) .
$$

Remark 14. Theorem 12 has links with classical analysis for second kind boundary equations on nonsmooth domains. Indeed, using the notation $\mathbf{x}^{j}=\left(x^{j}, y^{j}\right), D^{j, j \pm 1}$ can be written in layer-potential form as

$$
D^{j, j \pm 1} \psi^{j}(\mathbf{x})=-\frac{1}{\beta} \int_{\Sigma^{j}}\left(\alpha G\left(\mathbf{x}, \mathbf{x}^{j}\right)+\beta \frac{\partial G}{\partial x^{j \pm 1}}\left(\mathbf{x}, \mathbf{x}^{j}\right)\right) \psi^{j}\left(\mathbf{x}^{j}\right) \mathrm{d} \mathbf{x}^{j}, \quad \mathbf{x} \in \Sigma^{j \pm 1} \cap \Omega^{j},
$$

where $G$ is the Green's function for the Helmholtz equation satisfying the Robin condition on $\Sigma^{j}$ (see for instance [10] for a characterization of $G$ ). Formula (19) is nothing else but the Plancherel equality applied to (40). As $G$ is a smooth perturbation of the Green's function of the Laplace equation with Neumann boundary condition on $\Sigma^{j}$, and as $\operatorname{Im}(\omega)>0$, the properties of $D^{j, j \pm 1}$ can be deduced from those of the double layer potential operator defined by

$$
\int_{\Sigma^{j}} \frac{\partial G_{0}}{\partial x^{j \pm 1}}\left(\mathbf{x}, \mathbf{x}^{j}\right) \psi^{j}\left(\mathbf{x}^{j}\right) \mathrm{d} \mathbf{x}^{j}, \quad \mathbf{x} \in \Sigma^{j \pm 1} \cap \Omega^{j},
$$


where

$$
G_{0}\left(\mathbf{x}, \mathbf{x}^{\prime}\right)=\frac{1}{2 \pi} \log \left(\left|\mathbf{x}-\mathbf{x}^{\prime}\right|\right)
$$

This operator, as an operator acting on $L^{2}$ functions on the sides of a bounded polygon has been discussed and analyzed in [4,9]. Let us mention that in [9, Lemma 1], the same bound for the norm of the operator $L^{j, j \pm 1}$ has been found.

\subsection{Proof of Theorem 6}

Let us prove now that the operator $\mathbb{I}-\mathbb{D}$ is the sum of a coercive operator and a compact one in $\tilde{V}$. Using Theorem 12, and the following obvious decomposition

$$
\forall \tilde{\Phi}=\left(\tilde{\varphi}^{0}, \tilde{\varphi}^{1}, \ldots, \tilde{\varphi}^{n-1}\right) \in \tilde{V}, \quad \forall j \in \mathbb{Z} / n \mathbb{Z}, \tilde{\varphi}^{j}=\chi_{\left(-\infty, a_{j}^{-}\right)} \tilde{\varphi}^{j}+\chi_{\left(a_{j}^{+},+\infty\right)} \tilde{\varphi}^{j}
$$

we can decompose the operator $\mathbb{D}$ as follows

$$
\forall \tilde{\Phi} \in \tilde{V}, \quad \mathbb{D} \tilde{\Phi}=\mathbb{L} \tilde{\Phi}+\mathbb{K} \tilde{\Phi},
$$

where

$$
\mathbb{L}:=\left[\begin{array}{cccccc}
0 & L^{1,0} \chi_{\left(-\infty, a_{1}^{-}\right)} & 0 & \ldots & 0 & L^{n-1,0} \chi_{\left(a_{n-1}^{+},+\infty\right)} \\
L^{0,1} \chi_{\left(a_{0}^{+},+\infty\right)} & 0 & L^{2,1} \chi_{\left(-\infty, a_{2}^{-}\right)} & \ldots & 0 & 0 \\
0 & L^{1,2} \chi_{\left(a_{1}^{+},+\infty\right)} & 0 & \ldots & 0 & 0 \\
\vdots & \vdots & \vdots & \ddots & \vdots & \vdots \\
0 & 0 & 0 & \ldots & 0 & L^{n-1, n-2} \chi_{\left(-\infty, a_{n-1}^{-}\right)} \\
L^{0, n-1} \chi_{\left(-\infty, a_{0}^{-}\right)} & 0 & 0 & \ldots & L^{n-2, n-1} \chi_{\left(a_{n-2}^{+},+\infty\right)} & 0
\end{array}\right],
$$

and

$$
\mathbb{K}:=\left[\begin{array}{cccccc}
0 & K^{1,0} & 0 & \ldots & 0 & K^{n-1,0} \\
K^{0,1} & 0 & K^{2,1} & \ldots & 0 & 0 \\
0 & K^{1,2} & 0 & \ldots & 0 & 0 \\
\vdots & \vdots & \vdots & \ddots & \vdots & \vdots \\
0 & 0 & 0 & \ldots & 0 & K^{n-1, n-2} \\
K^{0, n-1} & 0 & 0 & \ldots & K^{n-2, n-1} & 0
\end{array}\right]
$$

with

$$
\forall j \in \mathbb{Z} / n \mathbb{Z}, \quad K^{j \pm 1, j}=\left(D^{j \pm 1, j}-L^{j \pm 1, j}\right)+L^{j \pm 1, j} \chi_{\left(a_{j \pm 1}^{ \pm}, \pm \infty\right)}
$$

From Theorem 12, we get easily that the operator $\mathbb{K}$ is compact in $\tilde{V}$.

Moreover, by Theorem 12 , for all $j \in \mathbb{Z} / n \mathbb{Z}$, we have that for all $\tilde{\varphi}^{j} \in L^{2}\left(\Sigma^{j}\right)$ such that $\left.\tilde{\varphi}^{j}\right|_{\Sigma_{\mathcal{O}}^{j}}=0$

$$
\begin{aligned}
\left\|L^{j, j-1} \chi_{\left(-\infty, a_{j}^{-}\right)} \tilde{\varphi}^{j}\right\|^{2}+\left\|L^{j, j+1} \chi_{\left(a_{j}^{+},+\infty\right)} \tilde{\varphi}^{j}\right\|^{2} & \leq C_{j}^{2}\left[\left\|\chi_{\left(-\infty, a_{j}^{-}\right)} \tilde{\varphi}^{j}\right\|^{2}+\left\|\chi_{\left(a_{j}^{+},+\infty\right)} \tilde{\varphi}^{j}\right\|^{2}\right] \\
& =C_{j}^{2}\left\|\tilde{\varphi}^{j}\right\|_{L^{2}\left(\Sigma^{j}\right)}^{2},
\end{aligned}
$$

where $C_{j}=\max \left(\cos \left(\theta^{j, j-1} / 2\right), \cos \left(\theta^{j, j+1} / 2\right)\right)$. Consequently the norm of the operator $\mathbb{L}$ is strictly less than 1 . This implies that the operator $\mathbb{I}-\mathbb{L}$ is coercive in $\tilde{V}$, its coercivity constant being given by

$$
\alpha=1-\max _{j \in \mathbb{Z} / n \mathbb{Z}} \cos \frac{\theta^{j, j+1}}{2} .
$$

Let us now show that Problem (33) is well-posed. Since it is Fredholm of index 0, it is sufficient to show the uniqueness. We will suppose that $\mathbb{F}=0$ and show that the corresponding solution $\tilde{\Phi} \in \tilde{V}$ necessarily vanishes. By Lemma 5, we can define unequivocally a function $p$ satisfying (14) for all $j \in \mathbb{Z} / n \mathbb{Z}$. Moreover, $p \in H^{1}(\Omega)$ and is solution of (1) with $g=0$. Problem (1) being well-posed, $p=0$ and then $P^{j}\left(\varphi^{j}\right)=0$ for all $j$. Consequently $\varphi^{j}=0$ for all $j \in \mathbb{Z} / n \mathbb{Z}$. 


\section{Discretization}

\subsection{The discrete problem}

To get a discrete problem that we can solve numerically, we use three main ingredients:

1. We truncate the integrals which appear in the definition of the integral operators $D^{j, j \pm 1}$ : the integral for $\xi \in \mathbb{R}$ is replaced by an integral for $|\xi| \leq \hat{T}$ for some $\hat{T} \in \mathbb{R}^{+}$.

2. Then, we introduce finite dimensional subspaces $\tilde{V}_{\mathbf{h}}$ of $\tilde{V}$ on which a Galerkin approximation is computed. To define the space $\tilde{V}_{\mathbf{h}}$, we truncate the infinite lines $\Sigma^{j}$ as follows:

$$
\Sigma_{T}^{j}=\left\{\left(x^{j}=l^{j}, y^{j}\right),-T_{j}<y^{j}<T_{j}\right\}
$$

and we mesh these truncated lines into segments $\left[M_{i}^{j}, M_{i+1}^{j}\right], i \in\left\{1, \ldots, N_{j}\right\}$ whose maximum length is $h_{j}$. Let $T=\min _{j} T_{j}$ and $h=\max _{j} h_{j}$. Finally, the space $V_{\mathbf{h}}$ with $\mathbf{h}=(T, h)$ built with Lagrange finite elements of degree $l\left(l \in \mathbb{N}^{*}\right)$ is given by:

$$
\left\{\left(\psi_{\mathbf{h}}^{0}, \ldots, \psi_{\mathbf{h}}^{n-1}\right) \in V, \quad \forall j, \psi_{\mathbf{h}}^{j} \text { is polynomial of degree } l \text { on }\left[M_{i}^{j}, M_{i+1}^{j}\right], i \in\left\{1, \ldots, N_{j}\right\}\right\},
$$

and $\tilde{V}_{\mathbf{h}}=V_{\mathbf{h}} \cap \tilde{V}$. Let us emphasize that of course

$$
\forall \tilde{\Psi} \in V, \quad \inf _{\psi_{\mathbf{h}} \in V_{\mathbf{h}}}\left\|\Psi-\Psi_{\mathbf{h}}\right\| \underset{\mathbf{h} \rightarrow(+\infty, 0)}{\longrightarrow} 0 .
$$

3. Finally, quadrature formulae have to be used to evaluate the Fourier integrals which appear in the variational formulation.

In what follows, we will study the error due to points 1 and 2 but not the quadrature formulae.

For this purpose, we consider the three following variational problems:

0 . The exact problem:

Find $\tilde{\Phi} \in \tilde{V}$ such that

$$
(\mathbb{B} \tilde{\Phi}, \tilde{\Psi})=(\mathbb{D} G, \tilde{\Psi}), \quad \forall \tilde{\Psi} \in \tilde{V}
$$

where $\mathbb{B}=\mathbb{I}-\mathbb{D}, \mathbb{D}$ is defined by $(25), \tilde{V}$ by $(26), G$ by $(30)$, and $(\cdot, \cdot)$ denotes the $L^{2}$ scalar product in $V$. We have the expression

$$
\forall(\Phi, \Psi) \in V, \quad(\mathbb{D} \Phi, \Psi)=\sum_{j \in \mathbb{Z} / n \mathbb{Z}}\left(\int_{\Sigma^{j} \cap \Omega^{j+1}}\left[D^{j+1, j} \varphi^{j+1}\right] \psi^{j}+\int_{\Sigma^{j} \cap \Omega^{j-1}}\left[D^{j-1, j} \varphi^{j-1}\right] \psi^{j}\right) .
$$

with

$$
\forall j \in \mathbb{Z} / n \mathbb{Z}, \quad \forall \psi \in L^{2}\left(\Sigma^{j}\right), \quad\left[D^{j \pm 1, j} \psi\right](r)=\frac{1}{\sqrt{2 \pi}} \int_{\mathbb{R}} k^{j \pm 1, j}(r, \xi) \hat{\psi}(\xi) \mathrm{d} \xi, \quad r \geq 0,
$$

and $k^{j \pm 1, j}(r, \xi)$ is given by $(20)$.

1. The semi-discrete problem (truncation of the integrals):

Find $\tilde{\Phi}_{\hat{T}} \in \tilde{V}$ such that

$$
\left(\mathbb{B}_{\hat{T}} \tilde{\Phi}_{\hat{T}}, \tilde{\Psi}\right)=\left(\mathbb{D}_{\hat{T}} G, \tilde{\Psi}\right), \quad \forall \tilde{\Psi} \in \tilde{V},
$$

where $\mathbb{B}_{\hat{T}}=\mathbb{I}-\mathbb{D}_{\hat{T}}$ and $\mathbb{D}_{\hat{T}}$ is defined by

$$
\forall(\Phi, \Psi) \in V, \quad\left(\mathbb{D}_{\hat{T}} \Phi, \Psi\right)=\sum_{j \in \mathbb{Z} / n \mathbb{Z}}\left(\int_{\Sigma^{j} \cap \Omega^{j+1}}\left[D_{\hat{T}}^{j+1, j} \varphi^{j+1}\right] \psi^{j}+\int_{\Sigma^{j} \cap \Omega^{j-1}}\left[D_{\hat{T}}^{j-1, j} \varphi^{j-1}\right] \psi^{j}\right),
$$

where

$$
\left[D_{\hat{T}}^{j \pm 1, j} \psi\right](r)=\frac{1}{\sqrt{2 \pi}} \int_{-\hat{T}}^{\hat{T}} k^{j \pm 1, j}(r, \xi) \hat{\psi}(\xi) \mathrm{d} \xi, \quad r \geq 0
$$


2. The discrete problem (truncation of the infinite lines $\Sigma^{j}$ and meshing):

Find $\tilde{\Phi}_{\hat{T}, \mathbf{h}} \in \tilde{V}_{\mathbf{h}}$ such that

$$
\left(\mathbb{B}_{\hat{T}} \tilde{\Phi}_{\hat{T}, \mathbf{h}}, \tilde{\Psi}_{\mathbf{h}}\right)=\left(\mathbb{D}_{\hat{T}} G_{\mathbf{h}}, \tilde{\Psi}_{\mathbf{h}}\right), \quad \forall \tilde{\Psi}_{\mathbf{h}} \in \tilde{V}_{\mathbf{h}},
$$

where $G_{\mathbf{h}} \in V_{\mathbf{h}}$ is the interpolate of $G$.

Our first objective is to prove that for $\hat{T}$ and $T$ large enough, and for $h$ small enough, the above discrete problem is well-posed. The second objective is to prove that the error $\left\|\Phi-\Phi_{\hat{T}, \mathbf{h}}\right\|$ (where $\Phi_{\hat{T}, \mathbf{h}}=\tilde{\Phi}_{\hat{T}, \mathbf{h}}+G_{\mathbf{h}}$ ) tends to 0 when $\hat{T} \rightarrow+\infty, T \rightarrow+\infty$, and $h \rightarrow 0$. And finally if the $\varphi^{j}$ 's are regular enough (whose precise definition will be given later), we will also estimate the convergence rate.

Remark 15. As in [18], the difficulty of the numerical analysis comes from the fact that the operator appearing in the discrete problem is the sum of a coercive part and a compact part, which both depend on $\hat{T}$.

As a first step, we will derive the same type of result but only for the semi-discrete problem.

\subsection{Numerical analysis of the semi-discrete problem}

For $\hat{T}>0$, we denote by $\Pi_{\hat{T}}$ the projection operator on $L^{2}(\mathbb{R})$ defined by

$$
\forall \psi \in L^{2}(\mathbb{R}), \quad \Pi_{\hat{T}} \psi(y)=\frac{1}{\sqrt{2 \pi}} \int_{-\hat{T}}^{\hat{T}} \hat{\psi}(\xi) e^{i \xi y} \mathrm{~d} \xi .
$$

In other words,

$$
\forall \psi \in L^{2}(\mathbb{R}), \quad \widehat{\Pi_{\hat{T}}}(\xi)=\chi_{[-\hat{T}, \hat{T}]}(\xi) \hat{\psi}(\xi) .
$$

Then, we denote by $\Pi_{\hat{T}}$ the projection operator on $V$ defined by

$$
\forall \Phi=\left(\varphi^{0}, \ldots, \varphi^{n-1}\right) \in V, \quad \Pi_{\hat{T}} \Phi=\left(\Pi_{\hat{T}} \varphi^{0}, \ldots, \Pi_{\hat{T}} \varphi^{n-1}\right) .
$$

Using Plancherel and Lebesgue theorems, one can easily check the following properties that will be used in the sequel:

$$
\begin{aligned}
\left\|\Pi_{\hat{T}} \Phi\right\|_{V} & \leq\|\Phi\|_{V} \\
\forall \Phi, \Psi \in V, \quad\left(\Pi_{\hat{T}} \Phi, \Psi\right) & =\left(\Phi, \Pi_{\hat{T}} \Psi\right) \\
\forall \Phi \in V, \quad\left\|\Pi_{\hat{T}} \Phi-\Phi\right\|_{V} & \rightarrow 0 \quad \text { when } \hat{T} \rightarrow+\infty
\end{aligned}
$$

Using this definition, we have $\mathbb{D}_{\hat{T}}=\mathbb{D} \Pi_{\hat{T}}$, where $D_{\hat{T}}$ is defined by (52). The main results of this section are given in the following theorem.

Theorem 16. 1. [Stability] There exists $\hat{T}_{\min }$ such that the semi-discrete problem (51) is wellposed for $\hat{T} \geq \hat{T}_{\min }$.

2. [Convergence] The solution $\tilde{\Phi}_{\hat{T}}$ of the semi-discrete problem (51) tends to the exact solution $\tilde{\Phi}$ of (49) when $\hat{T}$ tends to infinity.

3. [Error estimates] Let $\Phi=\tilde{\Phi}+G=\left(\varphi^{0}, \ldots, \varphi^{n-1}\right)$, where $\tilde{\Phi}$ is the solution of (49). If there exists $s>0$ such that for all $j \in \mathbb{Z} / n \mathbb{Z}, \varphi^{j} \in H^{s}\left(\Sigma^{j}\right)$, we have

$$
\left\|\tilde{\Phi}-\tilde{\Phi}_{\hat{T}}\right\|_{V} \leq \frac{C}{\hat{T}^{s}} \sum_{j \in \mathbb{Z} / n \mathbb{Z}}\left(\frac{1}{\sqrt{\sin \left(\theta^{j, j+1}\right)}}\left\|\varphi^{j+1}\right\|_{H^{s}\left(\Sigma^{j+1}\right)}+\frac{1}{\sqrt{\sin \left(\theta^{j, j-1}\right)}}\left\|\varphi^{j-1}\right\|_{H^{s}\left(\Sigma^{j-1}\right)}\right)
$$

The rest of this section is dedicated to the proof of this theorem. To do so, we will need several lemmas: 
- the two first lemmas give properties of the operators $L^{j \pm 1, j} \Pi_{\hat{T}}$ for the $L^{j \pm 1, j}$ appearing in (42). These results are the equivalent of the two properties stated in Theorem 12, but they are not a straightforward consequence of this theorem. The difficulty comes from the fact that, in general, for a function $\psi$, the support of $\Pi_{\hat{T}} \psi$ is not the same than the one of $\psi$. These lemmas enable us to deduce properties of the operator $\mathbb{D} \mathbb{\Pi}_{\hat{T}}$, used in Lemma 20 as a basic tool for the stability and the convergence result.

- To establish the error estimates, we will use finally Lemma 21.

Lemma 17. For all $j \in \mathbb{Z} / n \mathbb{Z}$, the operator $L^{j \pm 1, j}$ appearing in Theorem 12 satisfies

$$
\exists \tilde{C}^{j \pm 1, j} \in(0,1), \forall \varphi \in L^{2}\left(\Sigma^{j \pm 1}\right), \quad\left\|L^{j \pm 1, j} \Pi_{\hat{T}} \chi_{\left(a_{j \pm 1}^{\mp}, \mp \infty\right)} \varphi\right\| \leq \tilde{C}^{j \pm 1, j}\left\|\chi_{\left(a_{j \pm 1}^{\mp}, \mp \infty\right)} \varphi\right\| ;
$$

where $\tilde{C}^{j \pm 1, j}=\max \left(\sin \left(\theta^{j \pm 1, j} / 2\right), \cos \left(\theta^{j \pm 1, j} / 2\right)\right)$.

Proof. As explained in the proof of Lemma 11, $L^{0,1}=\chi_{(0, b)} L_{N}$ where $L_{N}$ is defined by (39). It suffices then to show that

$$
\forall \hat{T}>0, \forall \psi \in L^{2}\left(\Sigma^{0}\right) \quad\left\|L_{N} \Pi_{\hat{T}} \chi_{\left(a_{0}^{+},+\infty\right)} \psi\right\| \leq C\left\|\chi_{\left(a_{0}^{+},+\infty\right)} \psi\right\|
$$

with

$$
C=\max \left(\sin \left(\theta^{0,1} / 2\right), \cos \left(\theta^{0,1} / 2\right)\right)
$$

to obtain the result for $L^{0,1}$. A similar proof can be applied to other $L^{j \pm 1, j}$.

We stress again that (62) is not a direct consequence of Lemma 10 since $\Pi_{\hat{T}} \chi_{\left(a_{0}^{+},+\infty\right)} \psi$ is not supported in $\left(a_{0}^{+},+\infty\right)$.

We introduce the linear operators $S$ and $A$ of $\mathcal{L}\left(L^{2}(\mathbb{R})\right)$ defined by

$$
\forall \psi \in L^{2}(\mathbb{R}), \quad S \psi(y)=\frac{1}{2}\left(\psi(y)+\psi\left(2 a_{0}^{+}-y\right)\right) \quad \text { and } A \psi(y)=\frac{1}{2}\left(\psi(y)-\psi\left(2 a_{0}^{+}-y\right)\right)
$$

We have obviously $S+A=I d$.

The key point is that $\Pi_{\hat{T}}$ commutes with $S$ and $A$. Indeed, from

$$
\widehat{S \psi}(\xi) e^{\imath 2 a_{0}^{+} \xi}=\widehat{S \psi}(-\xi),
$$

we deduce that $\Pi_{\hat{T}} S \psi$ is symmetric with respect to $a_{0}^{+}$:

$$
\begin{aligned}
\Pi_{\hat{T}} S \psi\left(2 a_{0}^{+}-y\right) & =\frac{1}{\sqrt{2 \pi}} \int_{-\hat{T}}^{\hat{T}} \widehat{S \psi}(\xi) e^{i\left(2 a_{0}^{+}-y\right) \xi} \mathrm{d} \xi \\
& =\frac{1}{\sqrt{2 \pi}} \int_{-\hat{T}}^{\hat{T}} \widehat{S \psi}(-\xi) e^{-i y \xi} \mathrm{d} \xi \\
& =\frac{1}{\sqrt{2 \pi}} \int_{-\hat{T}}^{\hat{T}} \widehat{S \psi}(\xi) e^{i y \xi} \mathrm{d} \xi \\
& =\Pi_{\hat{T}} S \psi(y) .
\end{aligned}
$$

Similarly, we prove that $\Pi_{\hat{T}} A \psi$ is anti-symmetric with respect to $a_{0}^{+}$:

$$
\Pi_{\hat{T}} A \psi\left(2 a_{0}^{+}-y\right)=-\Pi_{\hat{T}} A \psi(y) .
$$

Finally, gathering all these properties, we get

$$
\begin{aligned}
S \Pi_{\hat{T}} \psi(y) & =\frac{1}{2}\left(\Pi_{\hat{T}} \psi(y)+\Pi_{\hat{T}} \psi\left(2 a_{0}^{+}-y\right)\right) \\
& =\frac{1}{2}\left(\Pi_{\hat{T}} S \psi(y)+\Pi_{\hat{T}} A \psi(y)+\Pi_{\hat{T}} S \psi\left(2 a_{0}^{+}-y\right)+\Pi_{\hat{T}} A \psi\left(2 a_{0}^{+}-y\right)\right) \\
& =\Pi_{\hat{T}} S \psi(y),
\end{aligned}
$$


and the same result can be obtained for $A$. To summarize, we have

$$
\Pi_{\hat{T}} S \psi=S \Pi_{\hat{T}} \psi \text { and } \Pi_{\hat{T}} A \psi=A \Pi_{\hat{T}} \psi .
$$

Now let us apply all these properties to our purpose. Since $S+A=I d, S^{2}=S$ and $A^{2}=A$ :

$$
\begin{aligned}
L_{N} \Pi_{\hat{T}} & =L_{N} \Pi_{\hat{T}}(S+A) \\
& =L_{N} \Pi_{\hat{T}}\left(S^{2}+A^{2}\right) \\
& =L_{N} S \Pi_{\hat{T}} S+L_{N} A \Pi_{\hat{T}} A
\end{aligned}
$$

so that

$$
\forall \psi \in L^{2}\left(\Sigma^{0}\right), \quad\left\|L_{N} \Pi_{\hat{T}} \chi_{\left(a_{0}^{+},+\infty\right)} \psi\right\| \leq\left\|L_{N} S\right\|\left\|\Pi_{\hat{T}}\right\|\left\|S \chi_{\left(a_{0}^{+},+\infty\right)} \psi\right\|+\left\|L_{N} A\right\|\left\|\Pi_{\hat{T}}\right\|\left\|A \chi_{\left(a_{0}^{+},+\infty\right)} \psi\right\| .
$$

Moreover, since for any $\psi \in L^{2}(\mathbb{R})$,

$$
\begin{aligned}
\left\|\Pi_{\hat{T}}\right\| & \leq 1 \text { and } \\
\left\|S \chi_{\left(a_{0}^{+},+\infty\right)} \psi\right\|_{L^{2}(\mathbb{R})} & =\left\|A \chi_{\left(a_{0}^{+},+\infty\right)} \psi\right\|_{L^{2}(\mathbb{R})}=\frac{1}{\sqrt{2}}\left\|\chi_{\left(a_{0}^{+},+\infty\right)} \psi\right\|_{L^{2}(\mathbb{R})},
\end{aligned}
$$

we get

$$
\left\|L_{N} \Pi_{\hat{T}} \chi_{\left(a_{0}^{+},+\infty\right)} \psi\right\| \leq \frac{1}{\sqrt{2}}\left(\left\|L_{N} S\right\|+\left\|L_{N} A\right\|\right)\left\|\chi_{\left(a_{0}^{+},+\infty\right)} \psi\right\|,
$$

Finally, the estimates (90) and (91) proven in the appendix enables us to show (62).

Remark 18. Let us emphasize that the key property (63) is true because in the definition of $\Pi_{\hat{T}}$, the Fourier integral has been truncated to a symmetric interval $[-\hat{T}, \hat{T}]$.

Lemma 19. For all $\hat{T}$, the operator $L^{j \pm 1, j} \Pi_{\hat{T}} \chi_{\left(a_{j \pm 1}^{ \pm}, \pm \infty\right)}$ is compact.

Moreover, let $\psi_{n}$ be a sequence of $L^{2}\left(\Sigma^{j \pm 1}\right)$, for $\hat{T}_{n} \rightarrow+\infty$ and for $j \in \mathbb{Z} / n \mathbb{Z}$ such that $\psi_{n}$ converges weakly to 0 in $L^{2}\left(\Sigma^{j \pm 1}\right)$, then

$$
L^{j \pm 1, j} \Pi_{\hat{T}_{n}} \chi_{\left(a_{j \pm 1}^{ \pm}, \pm \infty\right)} \psi_{n} \rightarrow 0 \quad \text { in } L^{2}\left(\Sigma^{j} \cap \Omega^{j \pm 1}\right) .
$$

Proof. As explained in the proof of the previous lemma, it suffices to show the result replacing $L^{j \pm 1, j}$ by $L_{N}$ to deduce the one for $L^{0,1}$ and in a similar way the one for the other $L^{j \pm 1, j}$.

Again, the difficulty is that, in general, for a function $\psi, \Pi_{\hat{T}} \chi_{\left(-\infty, a_{0}^{-}\right)} \psi$ is not supported in $\left(-\infty, a_{0}^{-}\right)$, so that the results cannot be deduced from the second point of Lemma 10.

We decompose

$$
L_{N} \Pi_{\hat{T}} \chi_{\left(-\infty, a_{0}^{-}\right)}=L_{N} \chi_{\left(-\infty, a_{0}^{-}\right)}+L_{N}\left(\Pi_{\hat{T}}-I\right) \chi_{\left(-\infty, a_{0}^{-}\right)}
$$

From Lemma 10 we know that $L_{N} \chi_{\left(-\infty, a_{0}^{-}\right)}$is compact. We show in the rest of the proof that $L_{N}\left(\Pi_{\hat{T}}-I\right) \chi_{\left(-\infty, a_{0}^{-}\right)}$is a Hilbert Schmidt operator and that $L_{N}\left(\Pi_{\hat{T}}-I\right) \chi_{\left(-\infty, a_{0}^{-}\right)} \rightarrow 0$ when $\hat{T} \rightarrow+\infty$, and the results of the lemma follow.

Using the expression of $L_{N}$ in (38), we get:

$$
L_{N}\left(\Pi_{\hat{T}}-I\right) \chi_{\left(-\infty, a_{0}^{-}\right)}=R_{\hat{T}}^{+} e^{\imath \theta^{0,1}}+R_{\hat{T}}^{-} e^{-\imath \theta^{0,1}},
$$


where, for $\psi \in L^{2}\left(\Sigma^{0}\right)$

$$
\begin{aligned}
& R_{\hat{T}}^{+} \psi(r)=\frac{1}{2 \pi} \int_{\xi>\hat{T}} \mathrm{~d} \xi \int_{-\infty}^{a_{0}^{-}} \mathrm{d} y e^{-\xi r \sin \theta^{0,1}} e^{\imath \xi\left(a_{0}^{+}-y+r \cos \theta^{0,1}\right)} \psi(y), \\
& R_{\hat{T}}^{-} \psi(r)=\frac{1}{2 \pi} \int_{\xi<-\hat{T}} \mathrm{~d} \xi \int_{-\infty}^{a_{0}^{-}} \mathrm{d} y e^{\xi r \sin \theta^{0,1}} e^{\imath \xi\left(a_{0}^{+}-y+r \cos \theta^{0,1}\right)} \psi(y) .
\end{aligned}
$$

By Fubini's Theorem, we deduce that

$$
R_{\hat{T}}^{ \pm} \psi(r)=\frac{1}{2 \pi} \int_{-\infty}^{a_{0}^{-}} k_{\hat{T}}^{ \pm}(r, y) \psi(y) \mathrm{d} y
$$

with

$$
k_{\hat{T}}^{ \pm}(r, y)=\frac{e^{-\hat{T}\left(r \sin \theta^{0,1} \pm \imath\left(a_{0}^{+}-y+r \cos \theta^{0,1}\right)\right)}}{r \sin \theta^{0,1} \mp \imath\left(a_{0}^{+}-y+r \cos \theta^{0,1}\right)} .
$$

Since the denominator never vanishes for $r \geq 0$ and $y<a_{0}^{-}<a_{0}^{+}$, one can easily check that

$$
\int_{0}^{+\infty} \int_{-\infty}^{a_{0}^{-}}\left|k_{\hat{T}}^{ \pm}(r, y)\right|^{2} \mathrm{~d} y \mathrm{~d} r
$$

is finite and tends to 0 when $\hat{T} \rightarrow+\infty$.

From these two lemmas, we deduce the following result.

Lemma 20. For all $\hat{T}>0$, the operator $\mathbb{B}_{\hat{T}}$ is the sum of a coercive operator and a compact operator in $\tilde{V}$.

Moreover, there exists $\gamma>0$ and $\hat{T}_{\text {min }}$ such that for $\hat{T} \geq \hat{T}_{\text {min }}$

$$
\forall \tilde{\Phi} \in \tilde{V} \quad\left\|\mathbb{B}_{\hat{T}} \tilde{\Phi}\right\| \geq \gamma\|\tilde{\Phi}\|
$$

Proof. Let $\hat{T}>0$. Let us remind that $\mathbb{B}_{\hat{T}}=\mathbb{I}-\mathbb{D} \Pi_{\hat{T}}$. By the definitions (25) of $\mathbb{D}$ and (57) of $\Pi_{\hat{T}}$, the operator $\mathbb{D} \Pi_{\hat{T}}$ is nothing else but $(25)$ with the terms $D^{j \pm 1, j}$ replaced by $D^{j \pm 1, j} \Pi_{\hat{T}}$. Finally, as in (41-42-43), we have the decomposition

$$
\mathbb{B}_{\hat{T}}=\mathbb{I}-\mathbb{D} \Pi_{\hat{T}} \quad \text { where } \mathbb{D} \Pi_{\hat{T}}=\mathbb{L}_{\hat{T}}+\mathbb{K}_{\hat{T}}
$$

with

- $\mathbb{L}_{\hat{T}}$ having the form of $(42)$ where the terms $L^{j \pm 1, j} \chi_{\left(a_{j \pm 1}^{\mp} \mp \infty\right)}$ are replaced by $L^{j \pm 1, j} \Pi_{\hat{T}} \chi_{\left(a_{j \pm 1}^{\mp}, \mp \infty\right)}$. Using Lemma 17 and the same arguments as in Section 3.2, we can show that the norm of $\mathbb{L}_{\hat{T}}$ is strictly less than 1 , the norm being independent of $\hat{T}$. Therefore $\mathbb{I}-\mathbb{L}_{\hat{T}}$ is coercive in $\tilde{V}$ with a coercive constant $\tilde{\gamma}$ independent of $\hat{T}$.

- $\mathbb{K}_{\hat{T}}$ has the form of (43) where the terms $K^{j \pm 1, j}$ are replaced by $K_{\hat{T}}^{j \pm 1, j}$ with

$$
K_{\hat{T}}^{j \pm 1, j}=\left(D^{j \pm 1, j}-L^{j \pm 1, j}\right) \Pi_{\hat{T}}+L^{j \pm 1, j} \Pi_{\hat{T}} \chi_{\left(a_{j \pm 1}^{ \pm}, \pm \infty\right)} \cdot
$$

By using Theorem 12 and Lemma $19, K_{\hat{T}}^{j \pm 1, j}$ is compact. The operator $\mathbb{K}_{\hat{T}}$ is then also compact in $\tilde{V}$.

We have then proven the first part of the theorem. We show the second part of the theorem by contradiction. We suppose the existence of a sequence $\tilde{\Phi}_{n} \in \tilde{V}$ and a sequence $\hat{T}_{n} \rightarrow+\infty$ such that $\left\|\tilde{\Phi}_{n}\right\|_{V}=1$ and $\mathbb{B}_{\hat{T}_{n}} \tilde{\Phi}_{n} \rightarrow 0$ in $\tilde{V}$. Using the first part of the proof, we have

$$
\mathbb{B}_{\hat{T}_{n}}=\left(\mathbb{I}-\mathbb{L}_{\hat{T}_{n}}\right)-\mathbb{K}_{\hat{T}_{n}},
$$


where the operator $\left(\mathbb{I}-\mathbb{L}_{\hat{T}_{n}}\right)$ is coercive with a coercivity constant $\tilde{\gamma}$ independent of $n$ and $\mathbb{K}_{\hat{T}_{n}}$ is compact. Rearranging the terms and taking the scalar product, we have

$$
\left(\mathbb{B}_{\hat{T}_{n}} \tilde{\Phi}_{n}, \tilde{\Phi}_{n}\right)+\left(\mathbb{K}_{\hat{T}_{n}} \tilde{\Phi}_{n}, \tilde{\Phi}_{n}\right)=\left(\left(\mathbb{I}-\mathbb{L}_{\hat{T}_{n}}\right) \tilde{\Phi}_{n}, \tilde{\Phi}_{n}\right) \geq \tilde{\gamma}>0,
$$

and we will show that left hand side tends to 0 with $n$ to establish the contradiction.

Since $\tilde{\Phi}_{n}$ is bounded in the Hilbert space $\tilde{V}$, it admits a weakly convergent subsequence that we denote also by $\tilde{\Phi}_{n}: \tilde{\Phi}_{n} \rightarrow \tilde{\Phi}$ in $\tilde{V}$. By (59) and (60),

$$
\forall \tilde{\Psi} \in \tilde{V}, \quad\left(\Pi_{\hat{T}_{n}} \tilde{\Phi}_{n}, \tilde{\Psi}\right)=\left(\tilde{\Phi}_{n}, \Pi_{\hat{T}_{n}} \tilde{\Psi}-\tilde{\Psi}\right)+\left(\tilde{\Phi}_{n}, \tilde{\Psi}\right) \rightarrow(\tilde{\Phi}, \tilde{\Psi}),
$$

which means that $\Pi_{\hat{T}_{n}} \tilde{\Phi}_{n} \rightarrow \tilde{\Phi}$. As a consequence,

$$
\mathbb{B}_{\hat{T}_{n}} \tilde{\Phi}_{n}=\tilde{\Phi}_{n}-\mathbb{D} \Pi_{\hat{T}_{n}} \tilde{\Phi}_{n} \rightarrow \tilde{\Phi}-\mathbb{D} \tilde{\Phi}=\mathbb{B} \tilde{\Phi}
$$

Since by hypothesis, $\mathbb{B}_{\hat{T}_{n}} \tilde{\Phi}_{n} \rightarrow 0$, we conclude that $\mathbb{B} \tilde{\Phi}=0$ which implies $\tilde{\Phi}=0$ because $\mathbb{B}$ is invertible (see Theorem 6).

On the other hand, as written in (69), each operator $K_{\hat{T}_{n}}^{j \pm 1, j}$ involved in the definition of $\mathbb{K}_{\hat{T}_{n}}$ is the sum of two operators such that

- $\left(D^{j \pm 1, j}-L^{j \pm 1, j}\right) \Pi_{\hat{T}_{n}} \tilde{\varphi}_{n} \rightarrow 0$ when $\tilde{\varphi}_{n} \rightarrow 0$ since $\Pi_{\hat{T}_{n}} \tilde{\varphi}_{n} \rightarrow 0$ because of $(60)$ and $\left(D^{j \pm 1, j}-L^{j \pm 1, j}\right)$ is compact because of Theorem 12;

- $L^{j \pm 1, j} \Pi_{\hat{T}_{n}} \chi_{\left(a_{j \pm 1}^{ \pm}, \pm \infty\right)} \tilde{\varphi}_{n} \rightarrow 0$ when $\tilde{\varphi}_{n} \rightarrow 0$ because of Lemma 19 .

Consequently, as $\tilde{\Phi}_{n} \rightarrow 0$, we have $\mathbb{K}_{\hat{T}_{n}} \tilde{\Phi}_{n} \rightarrow 0$ in $\tilde{V}$ when $n$ tends to $+\infty$.

Gathering all these results, we have $\left(\mathbb{B}_{\hat{T}_{n}} \tilde{\Phi}_{n}, \tilde{\Phi}_{n}\right)+\left(\mathbb{K}_{\hat{T}_{n}} \tilde{\Phi}_{n}, \tilde{\Phi}_{n}\right)$ tends to 0 with $n$. This contradiction finishes the proof.

To establish the error estimates of Theorem 16, we need the following lemma.

Lemma 21. Let $s>0$ and $\psi \in H^{s}\left(\Sigma^{j}\right)$. There exists a constant $C>0$ independent of $\psi$ and $\hat{T}$ such that

$$
\left\|D^{j, j \pm 1}\left(I-\Pi_{\hat{T}}\right) \psi\right\|_{L^{2}} \leq \frac{C}{\hat{T}^{s} \sqrt{\sin \left(\theta^{j, j \pm 1}\right)}}\|\psi\|_{H^{s}\left(\Sigma^{j}\right)}
$$

Proof. By definition (19-20) of $D^{j, j \pm 1}$, we have, by Cauchy-Schwarz inequality, Fubini's Theorem, and by the Fourier definition of the Sobolev spaces [2]:

$$
\left\|D^{j, j \pm 1}\left(I-\Pi_{\hat{T}}\right) \psi\right\|^{2} \leq\|\psi\|_{H^{s}\left(\Sigma^{j}\right)}^{2} \int_{|\xi|>\hat{T}} \int_{0}^{+\infty} \frac{\left|k^{j, j \pm 1}(r, \xi)\right|^{2}}{\left(1+\xi^{2}\right)^{s}} \mathrm{~d} \xi \mathrm{d} r
$$

Moreover, an easy calculation gives

$$
\int_{|\xi|>\hat{T}} \int_{0}^{+\infty} \frac{\left|k^{j, j \pm 1}(r, \xi)\right|^{2}}{\left(1+\xi^{2}\right)^{s}} \mathrm{~d} \xi \mathrm{d} r=\int_{|\xi|>\hat{T}} F(\xi) \mathrm{d} \xi
$$

where

$$
F(\xi)=\frac{\left|\alpha+\beta\left(-\cos \left(\theta^{j, j \pm 1}\right) i \sqrt{\omega^{2}-\xi^{2}}+\sin \left(\theta^{j, j \pm 1}\right) i \xi\right)\right|^{2}}{2\left|\alpha+i \beta \sqrt{\omega^{2}-\xi^{2}}\right|^{2} \operatorname{Im}\left(\sqrt{\omega^{2}-\xi^{2}}\right) \sin \left(\theta^{j, j \pm 1}\right)\left(1+\xi^{2}\right)^{s}}
$$

is such that

$$
F(\xi) \leq \frac{C}{\xi^{2 s+1} \sin \left(\theta^{j, j \pm 1}\right)}
$$

for some constant $C$ depending only on $\alpha, \beta, \omega$, and $s$. The result follows. 


\section{Proof of Theorem 16.}

1. By Lemma $20, \mathbb{B}_{\hat{T}}$ is the sum of a coercive and a compact operators. By Fredholm alternative, it is invertible if and only if it is injective. Again by lemma 20, we have that there exists $\hat{T}_{\text {min }}$ such that for $\hat{T} \geq \hat{T}_{\min }, \mathbb{B}_{\hat{T}}$ is injective.

2. From $\mathbb{B} \tilde{\Phi}=\mathbb{D} G$ and $\mathbb{B}_{\hat{T}} \tilde{\Phi}_{\hat{T}}=\mathbb{D} \Pi_{\hat{T}} G$, we deduce:

$$
\begin{aligned}
\mathbb{B}_{\hat{T}}\left(\tilde{\Phi}-\tilde{\Phi}_{\hat{T}}\right) & =\mathbb{B} \tilde{\Phi}-\left(\mathbb{B}-\mathbb{B}_{\hat{T}}\right) \tilde{\Phi}-\mathbb{B}_{\hat{T}} \tilde{\Phi}_{\hat{T}} \\
& =\mathbb{D}\left(\mathbb{I}-\mathbb{\Pi}_{\hat{T}}\right)(\tilde{\Phi}+G) \\
& =\mathbb{D}\left(\mathbb{I}-\mathbb{\Pi}_{\hat{T}}\right) \Phi
\end{aligned}
$$

which tends to 0 when $\hat{T}$ tends to $+\infty$ by (60). Lemma 20 then implies that

$$
\left\|\tilde{\Phi}-\tilde{\Phi}_{\hat{T}}\right\| \leq \frac{1}{\gamma}\left\|\mathbb{B}_{\hat{T}}\left(\tilde{\Phi}-\tilde{\Phi}_{\hat{T}}\right)\right\|
$$

which proves that $\tilde{\Phi}_{\hat{T}}$ tends to $\tilde{\Phi}$ when $\hat{T}$ tends to $+\infty$.

3. The previous step provides also the following inequality:

$$
\left\|\tilde{\Phi}-\tilde{\Phi}_{\hat{T}}\right\| \leq \frac{1}{\gamma}\left\|\mathbb{D}\left(\mathbb{I}-\Pi_{\hat{T}}\right) \Phi\right\|
$$

Combined with Lemma 21, we get the estimate (61).

\subsection{Error estimate for the discrete problem}

The main result of this section is given in the following theorem.

Theorem 22. 1. There exist $\hat{T}_{\min }, T_{\min }$ and $h_{\max }$ such that the discrete problem (54) is well-posed for $\hat{T} \geq \hat{T}_{\min }, T \geq T_{\min }$ and $h \leq h_{\max }$.

2. The solution $\tilde{\Phi}_{\hat{T}, \mathbf{h}}$ of the discrete problem (54) tends to the exact solution $\tilde{\Phi}$ of (49) when $\hat{T} \rightarrow+\infty$ and $\mathbf{h}=(T, h) \rightarrow(+\infty, 0)$.

3. If $\Phi=\tilde{\Phi}+G$ is such that $\varphi^{j} \in H^{s}\left(\Sigma^{j}\right)$ for $j \in \mathbb{Z} / n \mathbb{Z}$ with $s>0$, there exists $C>0$ such that

$$
\left\|\tilde{\Phi}-\tilde{\Phi}_{\hat{T}, \mathbf{h}}\right\| \leq \frac{C}{\hat{T}^{s}}+C e^{-\varepsilon T}+C h^{\min (s, l+1)}
$$

where $\varepsilon$ is given by $(3)$.

To show this theorem, we will use the following lemma (which is the discrete equivalent of Lemma $20)$.

Lemma 23. There exists $\gamma^{\prime}>0, \hat{T}_{\min }, T_{\min }$ and $h_{\max }$ such that for $\hat{T} \geq \hat{T}_{\min }, T \geq T_{\min }$ and $h \leq h_{\max }$,

$$
\forall \tilde{\Phi}_{\mathbf{h}} \in \tilde{V}_{\mathbf{h}} \sup _{\tilde{\Psi}_{\mathbf{h}} \in \tilde{V}_{\mathbf{h}}, \tilde{\Psi}_{\mathbf{h}} \neq 0} \frac{\left|\left(\mathbb{B}_{\hat{T}} \tilde{\Phi}_{\mathbf{h}}, \tilde{\Psi}_{\mathbf{h}}\right)\right|}{\left\|\tilde{\Psi}_{\mathbf{h}}\right\|} \geq \gamma^{\prime}\left\|\tilde{\Phi}_{\mathbf{h}}\right\| .
$$

Proof. We proceed as in the proof of Lemma 20 and prove the result by contradiction. We consider a sequence $h_{n}, h_{n} \rightarrow 0$, a sequence $T_{n}, T_{n} \rightarrow+\infty$, a sequence $\hat{T}_{n}, \hat{T}_{n} \rightarrow+\infty$, and a sequence $\tilde{\Phi}_{\hat{T}_{n}, \mathbf{h}_{n}} \in$ $\tilde{V}_{\hat{T}_{n}, \mathbf{h}_{n}}, \mathbf{h}_{n}=\left(h_{n}, T_{n}\right)$ such that

$$
\left\|\tilde{\Phi}_{\hat{T}_{n}, \mathbf{h}_{n}}\right\|=1 \quad \text { and } \quad \forall \tilde{\Psi}_{\mathbf{h}_{n}} \in \tilde{V}_{\mathbf{h}_{n}},\left|\left(\mathbb{B}_{\hat{T}_{n}} \tilde{\Phi}_{\hat{T}_{n}, \mathbf{h}_{n}}, \tilde{\Psi}_{\mathbf{h}_{n}}\right)\right| \leq \frac{1}{n}\left\|\tilde{\Psi}_{\mathbf{h}_{n}}\right\| .
$$


Since $\tilde{\Phi}_{\hat{T}_{n}, \mathbf{h}_{n}}$ is bounded in $\tilde{V}$, it admits a weakly convergent subsequence that we denote also by $\tilde{\Phi}_{\hat{T}_{n}, \mathbf{h}_{n}}: \tilde{\Phi}_{\hat{T}_{n}, \mathbf{h}_{n}} \rightarrow \tilde{\Phi}$. Moreover, for all $\tilde{\Psi} \in \tilde{V}$ and all $\tilde{\Psi}_{\mathbf{h}_{n}} \in \tilde{V}_{\mathbf{h}_{n}}$ we have

$$
\begin{aligned}
\left|\left(\mathbb{B}_{\hat{T}_{n}} \tilde{\Phi}_{\hat{T}_{n}, \mathbf{h}_{n}}, \tilde{\Psi}\right)\right| & \leq\left|\left(\mathbb{B}_{\hat{T}_{n}} \tilde{\Phi}_{\hat{T}_{n}, \mathbf{h}_{n}}, \tilde{\Psi}_{\mathbf{h}_{n}}\right)\right|+\left|\left(\mathbb{B}_{\hat{T}_{n}} \tilde{\Phi}_{\hat{T}_{n}, \mathbf{h}_{n}}, \tilde{\Psi}_{-} \tilde{\Psi}_{\mathbf{h}_{n}}\right)\right| \\
& \leq \frac{1}{n}\left\|\tilde{\Psi}_{\mathbf{h}_{n}}\right\|+\left\|\mathbb{B}_{\hat{T}_{n}}\right\|\left\|\tilde{\Psi}-\tilde{\Psi}_{\mathbf{h}_{n}}\right\| .
\end{aligned}
$$

Since $\left\|\mathbb{B}_{\hat{T}_{n}}\right\|$ is bounded by a constant independent of $n$, we deduce from (48) that

$$
\mathbb{B}_{\hat{T}_{n}} \tilde{\Phi}_{\hat{T}_{n}, \mathbf{h}_{n}} \rightarrow 0 \quad \text { in } \tilde{V}
$$

We can then continue the proof as in Lemma 20 which results in the contradiction.

\section{Proof of Theorem 22.}

1. This is a direct consequence of Lemma 23.

2. Let $\tilde{\Phi}$ be the solution of the original problem (49), $\tilde{\Phi}_{\hat{T}}$ the solution of the semi discrete problem (51) and $\tilde{\Phi}_{\hat{T}, \mathbf{h}}$ the solution of the discrete problem (54). We have that

$$
\forall \tilde{\Upsilon}_{\mathbf{h}} \in \tilde{V}_{\mathbf{h}}, \quad\left\|\tilde{\Phi}-\tilde{\Phi}_{\hat{T}, \mathbf{h}}\right\| \leq\left\|\tilde{\Phi}-\tilde{\Upsilon}_{\mathbf{h}}\right\|+\left\|\tilde{\Upsilon}_{\mathbf{h}}-\tilde{\Phi}_{\hat{T}, \mathbf{h}}\right\|
$$

For all $\tilde{\Upsilon}_{\mathbf{h}} \in \tilde{V}_{\mathbf{h}}$ and all $\tilde{\Psi}_{\mathbf{h}} \in \tilde{V}_{\mathbf{h}}$, we have

$$
\left(\mathbb{B}_{\hat{T}}\left(\tilde{\Upsilon}_{\mathbf{h}}-\tilde{\Phi}_{\hat{T}, \mathbf{h}}\right), \tilde{\Psi}_{\mathbf{h}}\right)=\left(\mathbb{B}_{\hat{T}}\left(\tilde{\Upsilon}_{\mathbf{h}}-\tilde{\Phi}_{\hat{T}}\right), \tilde{\Psi}_{\mathbf{h}}\right)+\left(\mathbb{D}_{\hat{T}}\left(G-G_{\mathbf{h}}\right), \tilde{\Psi}_{\mathbf{h}}\right) .
$$

By Lemma 23 and by the continuity of $\mathbb{D}_{\hat{T}}$ and $\mathbb{B}_{\hat{T}}$, we get

$$
\gamma^{\prime}\left\|\tilde{\Upsilon}_{\mathbf{h}}-\tilde{\Phi}_{\hat{T}, \mathbf{h}}\right\| \leq C\left(\left\|G-G_{\mathbf{h}}\right\|_{V}+\left\|\tilde{\Phi}_{\hat{T}}-\tilde{\Upsilon}_{\mathbf{h}}\right\|\right) .
$$

Gathering (75-76), we deduce that there exists $C>0$, such that

$$
\left\|\tilde{\Phi}-\tilde{\Phi}_{\hat{T}, \mathbf{h}}\right\| \leq C\left(\left\|\tilde{\Phi}-\tilde{\Phi}_{\hat{T}}\right\|+\left\|G-G_{\mathbf{h}}\right\|_{V}+\inf _{\tilde{\Upsilon}_{\mathbf{h}} \in \tilde{V}_{\mathbf{h}}, \tilde{\Upsilon}_{\mathbf{h}} \neq 0}\left\|\tilde{\Phi}-\tilde{\Upsilon}_{\mathbf{h}}\right\|\right) .
$$

By Theorem 16, the first term of the right hand side tends to $0 . G_{\mathbf{h}}$ being the interpolant of $G$ in $\tilde{V}_{\mathbf{h}},(48)$ ensures that the two last terms tend to 0 when $\mathbf{h} \rightarrow(+\infty, 0)$.

3. Let now suppose that $\Phi=\tilde{\Phi}+G=\left(\varphi^{0}, \ldots, \varphi^{n-1}\right)$ the solution of (49) is such that for all $j \in \mathbb{Z} / n \mathbb{Z}, \varphi^{j} \in H^{s}\left(\Sigma^{j}\right)$ for a certain $s>0$. Then we deduce from Theorem 16 an estimation of the first term of the right hand side of (77). For the second term, it suffices to use classical results of the interpolation error for Lagrange FE of order $l$ :

$$
\exists C>0, \quad\left\|G-G_{\mathbf{h}}\right\|_{V} \leq C h^{\min (s, l+1)} .
$$

Finally for the last term, let us introduce the function $\tilde{\Phi}_{T} \in \tilde{V}$ defined by

$$
\tilde{\Phi}_{T}=\Phi_{T}-G \quad \text { where } \Phi_{T}=\left(\chi_{\left(-T_{0}, T_{0}\right)} \varphi^{0}, \ldots, \chi_{\left(-T_{n-1}, T_{n-1}\right)} \varphi^{n-1}\right) .
$$

We get

$$
\inf _{\tilde{\Upsilon}_{\mathbf{h}} \in \tilde{V}_{\mathbf{h}}, \tilde{\Upsilon}_{\mathbf{h}} \neq 0}\left\|\tilde{\Phi}-\tilde{\Upsilon}_{\mathbf{h}}\right\| \leq\left\|\tilde{\Phi}-\tilde{\Phi}_{T}\right\|+\inf _{\tilde{\Upsilon}_{\mathbf{h}} \in \tilde{V}_{\mathbf{h}}, \tilde{\Upsilon}_{\mathbf{h}} \neq 0}\left\|\tilde{\Phi}_{T}-\tilde{\Upsilon}_{\mathbf{h}}\right\|
$$

where using (3), we can show that

$$
\left\|\tilde{\Phi}-\tilde{\Phi}_{T}\right\| \leq C e^{-\varepsilon T}
$$

and using again the results on interpolation error of Lagrange FE

$$
\inf _{\tilde{\Upsilon}_{\mathbf{h}} \in \tilde{V}_{\mathbf{h}}, \tilde{\Upsilon}_{\mathbf{h}} \neq 0}\left\|\tilde{\Phi}_{T}-\tilde{\Upsilon}_{\mathbf{h}}\right\| \leq C h^{\min (s, l+1)} .
$$

This ends the proof of the theorem.

Remark 24. This error estimate has been obtained for simple regular mesh. A more sophisticated discretization method could be used as done in [11] for scattering problems. 


\section{$5 \quad$ Numerical results}

The numerical results presented in this section are obtained using the Finite Element library XLiFE++ [16].

\subsection{Qualitative validation of the method}

In order to validate the method, we consider a particular data on a triangle given by

$$
g=\left.\frac{1}{4 \imath}\left(\alpha H\left(\omega \sqrt{x^{2}+y^{2}}\right)+\beta \frac{\partial H}{\partial x^{j}}\left(\omega \sqrt{x^{2}+y^{2}}\right)\right)\right|_{\Sigma_{\mathcal{O}}^{j}}
$$

with $\omega=1+0.1 \imath, \alpha=2, \beta=-0.5$ and $H($.$) denotes the zeroth Hankel function of the first kind [3].$ The exact solution of this problem is

$$
p=\frac{1}{4 \imath} H\left(\omega \sqrt{x^{2}+y^{2}}\right) .
$$

On Figure 4 we represent on the interval $(-T, T)$ the real and imaginary parts of the exact solution $\varphi^{0}$ (blue line) and of the solution $\varphi_{\hat{T}, \mathbf{h}}^{0}$ (red dots) computed by using P1 finite elements with $h=0.1$, $T=20, \hat{T}=10$, and a 3 rd order Gauss quadrature with 1000 intervals. We get an $L^{2}$ relative error:

$$
\frac{\left\|\varphi^{0}-\varphi_{\hat{T}, \mathbf{h}}^{0}\right\|_{L^{2}\left(\Sigma_{T}^{0}\right)}}{\left\|\varphi^{0}\right\|_{L^{2}\left(\Sigma_{T}^{0}\right)}}
$$

of $0.090 \%$. On Figure 5 (left), we represent the Fourier transform of the computed solution. Remark that the behavior of this Fourier transform justifies the truncation of the Fourier integral and requires a precise quadrature especially near $\xi=\omega$.
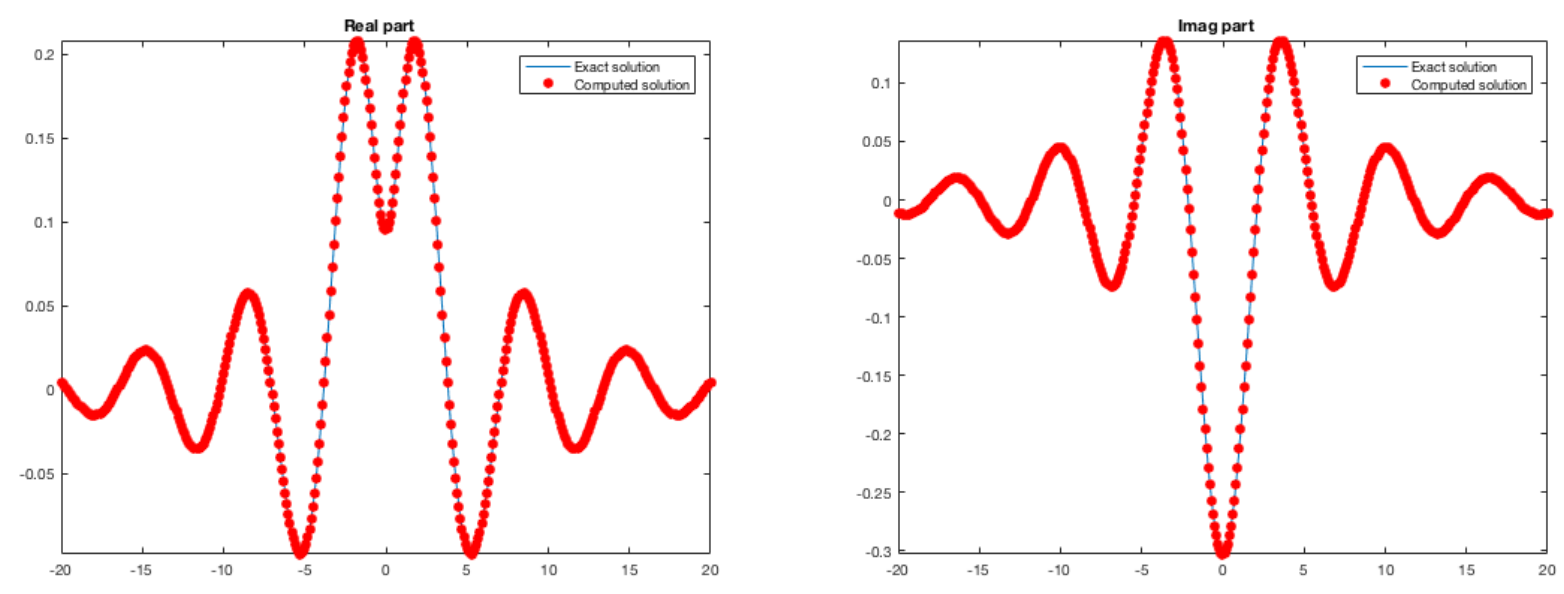

Figure 4: Real (left) and imaginary (right) part of the computed solution $\varphi_{\hat{T}, \mathbf{h}}^{0}$ (red points) and the exact solution (blue line) on $\Sigma^{0}$.

Once we obtained the $\varphi_{\hat{T}, \mathbf{h}}^{j}$ 's, we can reconstruct an approximation of the solution $p$ of (1) in each $\Omega^{j}$ by Formula (12). Here, we compute the solution in the domain $\Omega^{\prime}$ represented in Figure 5 (right), where the white lines represent the position of the $\Sigma^{j}$. In the overlapping zones, we can choose indifferently one of the available half-plane representations, since they coincide up to the discretization error. Remark that although the solutions $\varphi^{j}$ are not close to zero at $y^{j}= \pm T$, the reconstructed solution is accurate, with an $L^{2}\left(\Omega^{\prime}\right)$ relative error equal to $0.030 \%$. 

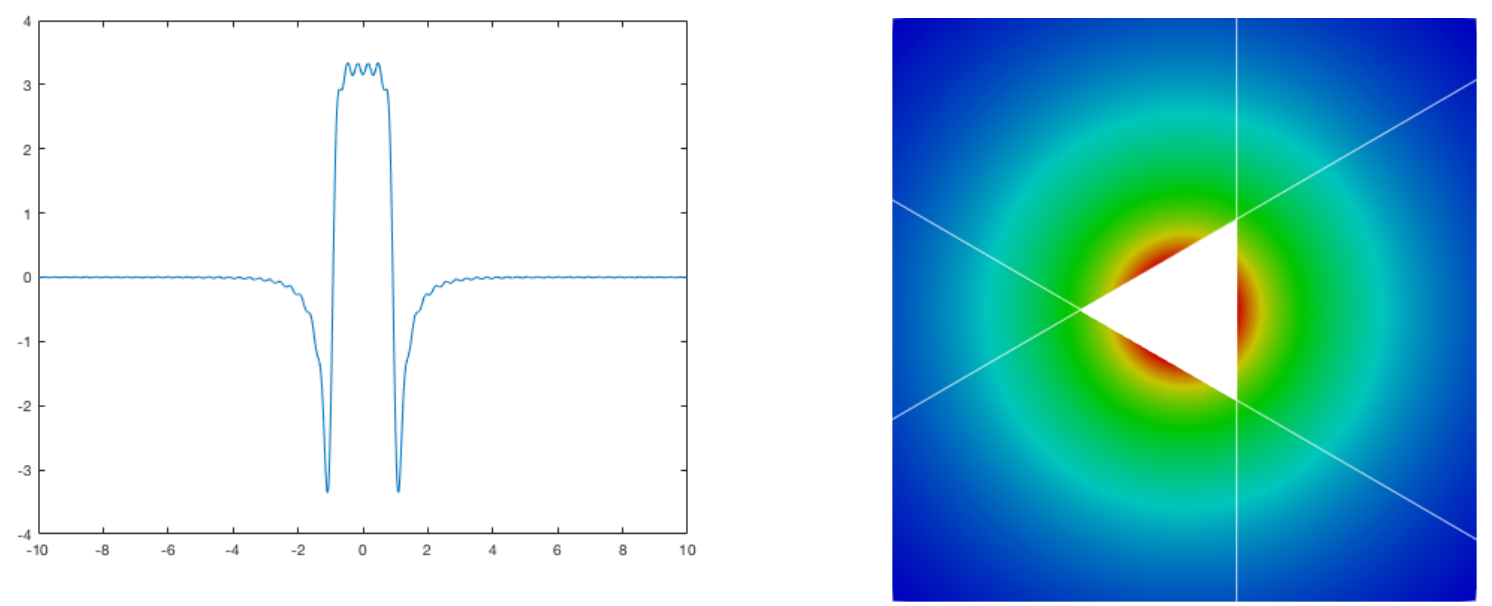

Figure 5: On the left: real part of the Fourier transform $\hat{\varphi}_{\hat{T}, \mathbf{h}}^{0}$. On the right: reconstruction of the solution in $\Omega^{\prime}$.

The same results can also be obtained when the obstacle is a rectangle or a pentagon. The reconstruction results are shown in Figure 6. For a rectangle obstacle, the $L^{2}$ relative error for the lines is $0.042 \%$ and the $L^{2}$ error on the reconstructed domain is $0.043 \%$, while for a pentagon, we get $0.074 \% L^{2}$ relative error on the lines and $0.054 \% L^{2}$ relative error on the reconstructed domain.
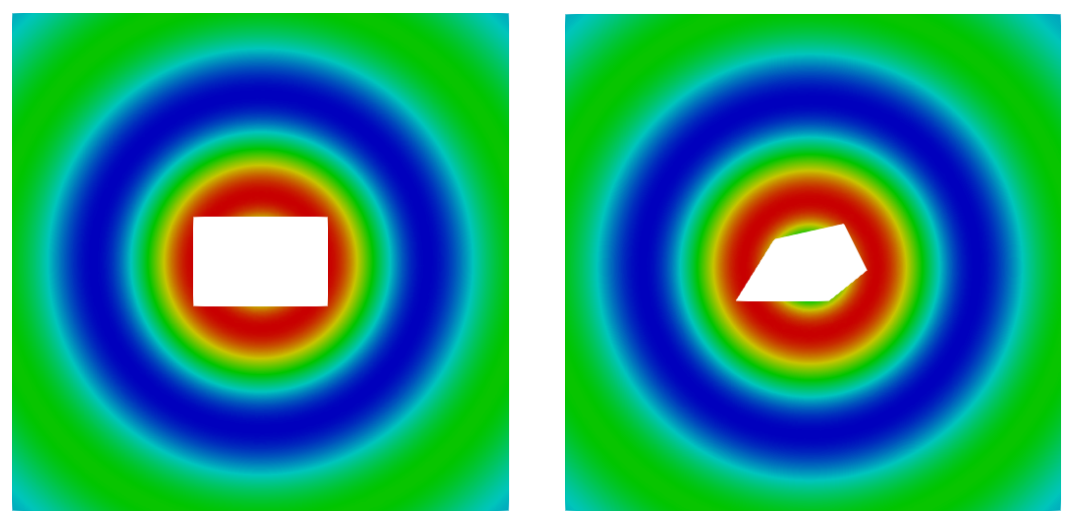

Figure 6: Reconstruction of the solution in $\Omega$ with rectangle and pentagon.

\subsection{Quantitative validation of the error estimation}

After this qualitative validation, we validate the error estimation derived in Section 4 by studying the influence of the different parameters. Since the triangle is regular, it suffices, by symmetry, to only consider the error on $\Sigma^{0}$. We still consider $\alpha=2, \beta=-0.5$, and except in Section 5.2.1, $\omega=1+0.1$.

\subsubsection{Influence of the length of the lines (parameter $T$ )}

From (74), we expect that the error will decay like $e^{-\varepsilon T}$, where $\varepsilon$ is the imaginary part of the frequency. That is why, in this section (and only in this section), we consider different values of $\varepsilon \in\{0.05,0.1,0.2\}$. We fix the other parameters to $h=0.025, \hat{T}=10$ and use a 3rd order Gauss quadrature with 1000 intervals. 


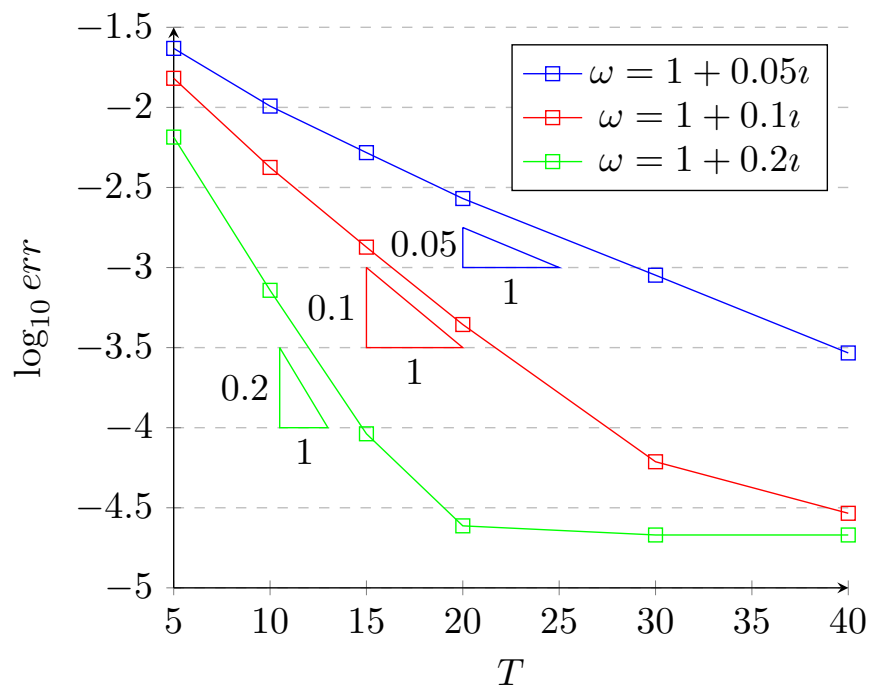

Figure 7: Influence of the length of the lines $T$ for various values of $\varepsilon=\operatorname{Im}(\omega)$.

In Figure 7, we represent $\log \left(\varphi^{0}-\varphi_{\hat{T}, \mathbf{h}}^{0}\right)$ as a function of $T$. The errors $\left(\varphi^{0}-\varphi_{\hat{T}, \mathbf{h}}^{0}\right)$ decrease exponentially, depending on $\varepsilon$ with the following behavior

$$
\operatorname{err}:=\left\|\varphi^{0}-\varphi_{T, \mathbf{h}}^{0}\right\|_{L^{2}\left(\Sigma_{T}^{0}\right)} \sim e^{-\varepsilon T},
$$

before finally becoming constant, which is due to the other discretization parameters.

\subsubsection{Influence of the discretization in space (parameter $h$ )}

We plot the error $\log \left(\varphi^{0}-\varphi_{\hat{T}, \mathbf{h}}^{0}\right)$ as a function of $\log h$. We use the P1 and P2 finite elements and the following parameters:

$$
T=40, \hat{T}=10,
$$

and a 3rd order quadrature with 1000 intervals.

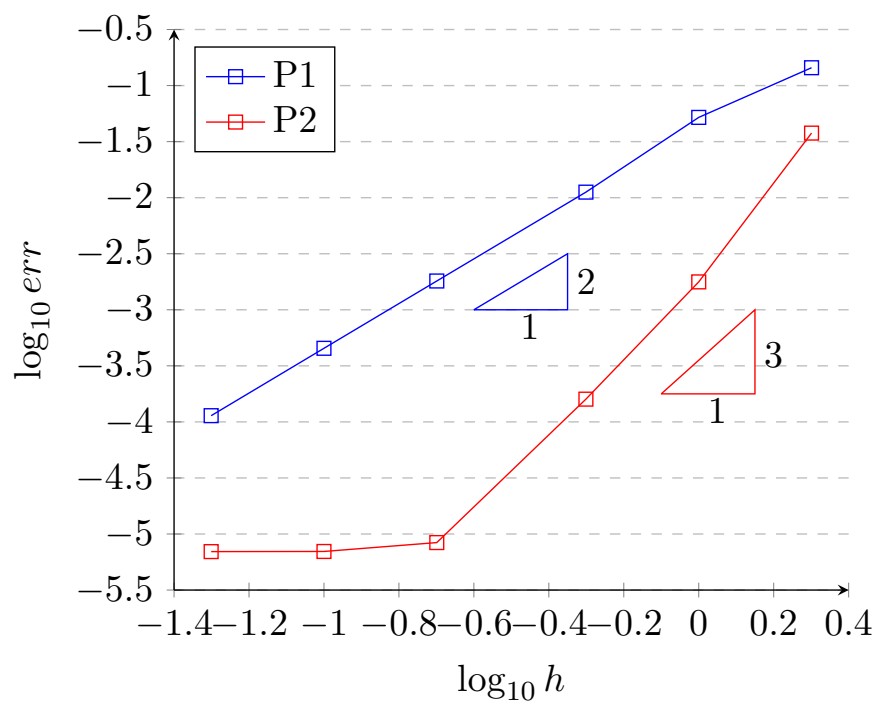

Figure 8: Influence of the space discretization $h$.

Figure 8 shows that the error decreases following

$$
\text { err } \sim h^{l+1},
$$

before becoming constant because of the other discretization parameters. 


\subsubsection{Influence of the truncation of the Fourier integrals (parameter $\hat{T}$ )}

Finally, we plot the error $\log \left(\varphi^{0}-\varphi_{\hat{T}, \mathbf{h}}^{0}\right)$ with respect to $\hat{T}$ and we use $T=40, h=0.1$, and a 3 rd order quadrature with $100 \times \hat{T}$ intervals.

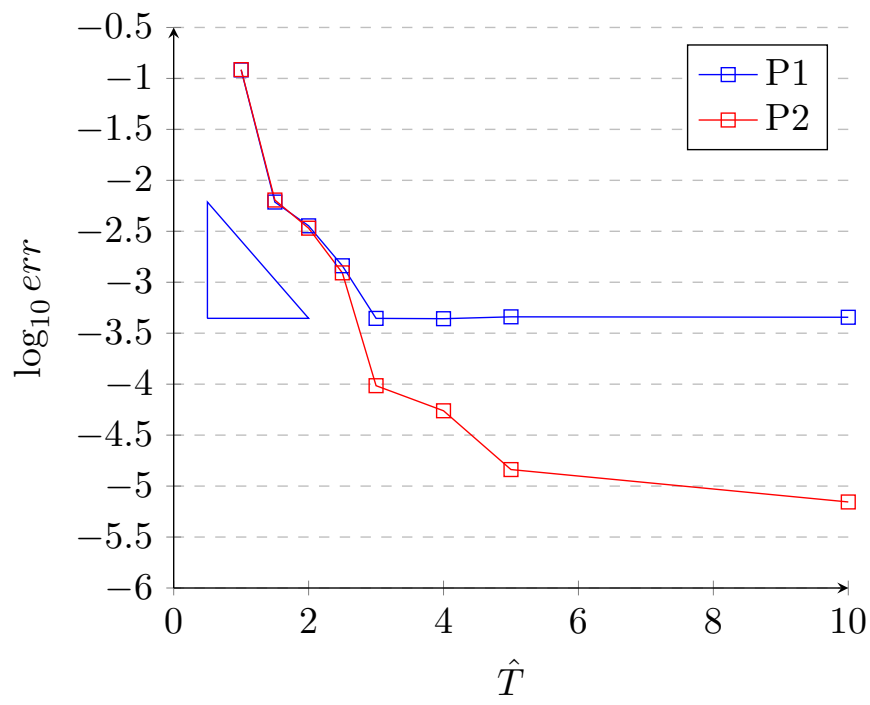

Figure 9: Influence of the length of the Fourier integral $\hat{T}$.

From Figure 9, we see that the error decreases exponentially due to the $\mathcal{C}^{\infty}$ regularity of the Hankel function.

\subsubsection{The influence of the angles of the polygon}

Refering to the Theorem 12, we investigate the influence of the angles of the polygon on the computation of the solution. Remember that the coercivity constant tends to zero when one of the angles tends to zero (see (45)).

We represent the reconstruction of the solution around three different triangles with one angle becoming smaller and smaller $\left(\min \left(\theta^{j, j+1}\right)=0.33 \pi, 0.16 \pi, 0.03 \pi\right)$. Qualitatively, the results look similar and the $L^{2}$ relative error are of the same order (respectively $1.01 \%, 0.88 \%$, and $1.23 \%$ ). The condition number of the finite element matrices are 1617.27, 2482.05, and 4647.19 respectively, meaning that it is only slightly affected by the smallness of one of the angles.
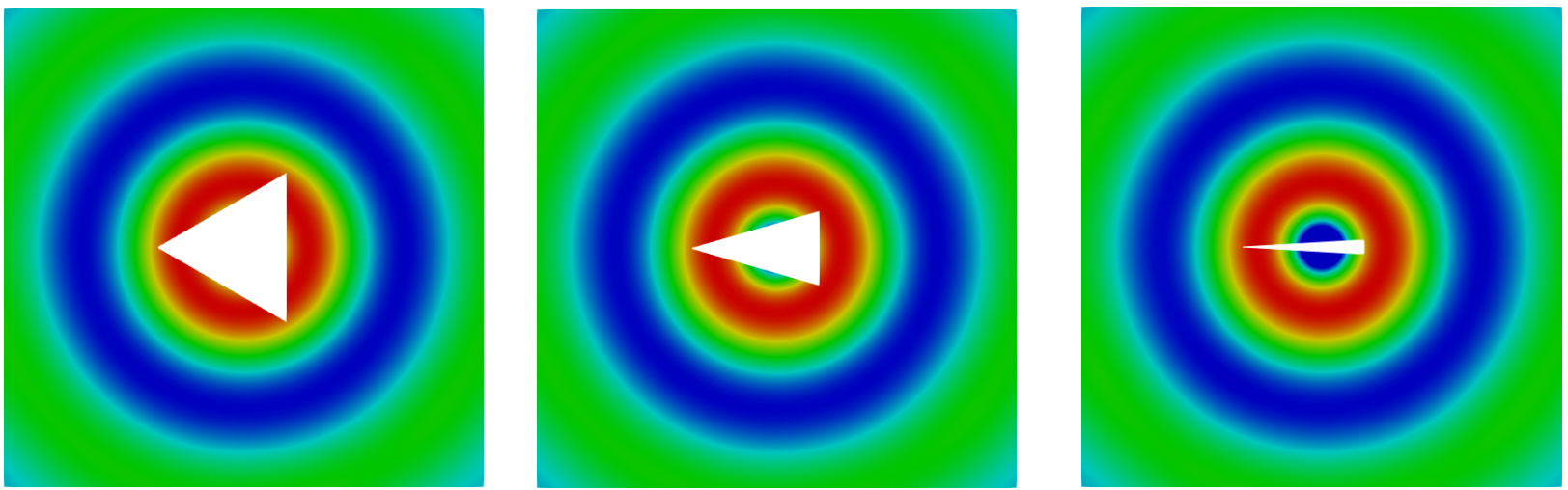

Figure 10: Reconstruction of the solution in $\Omega$ with triangles that becomes more and more flat. 


\subsection{Extension cases}

\subsubsection{Non-regular Dirichlet data}

In this section, we consider the Dirichlet case, namely (1) with $\alpha=1, \beta=0$ and we use the HalfSpace Matching formulation (22) where the $\varphi^{j}$ 's correspond to the Dirichlet traces of $p$ on the $\Sigma^{j}$ 's. As mentioned in Section 1.2, our formulation allows to consider a data $g \in L^{2}(\partial \mathcal{O})$ but $g \notin H^{1 / 2}(\partial \mathcal{O})$. In the following test, we take

$$
g= \begin{cases}1 & \text { if } x^{0}=0, y^{0}>0 \text { or } x^{1}=0, y^{1}<0 \\ 0 & \text { otherwise }\end{cases}
$$

We use P1 discontinuous finite elements since we have a discontinuous boundary condition on $\partial \mathcal{O}$. The real part of the $\varphi_{T, \mathbf{h}}^{0}$ and the Fourier transform are given in Figure 11. As the data is less regular than the previous example, the Fourier transform $\hat{\varphi}_{T, \mathbf{h}}^{0}$ decays more slowly than in the previous example (pay attention to the scale).
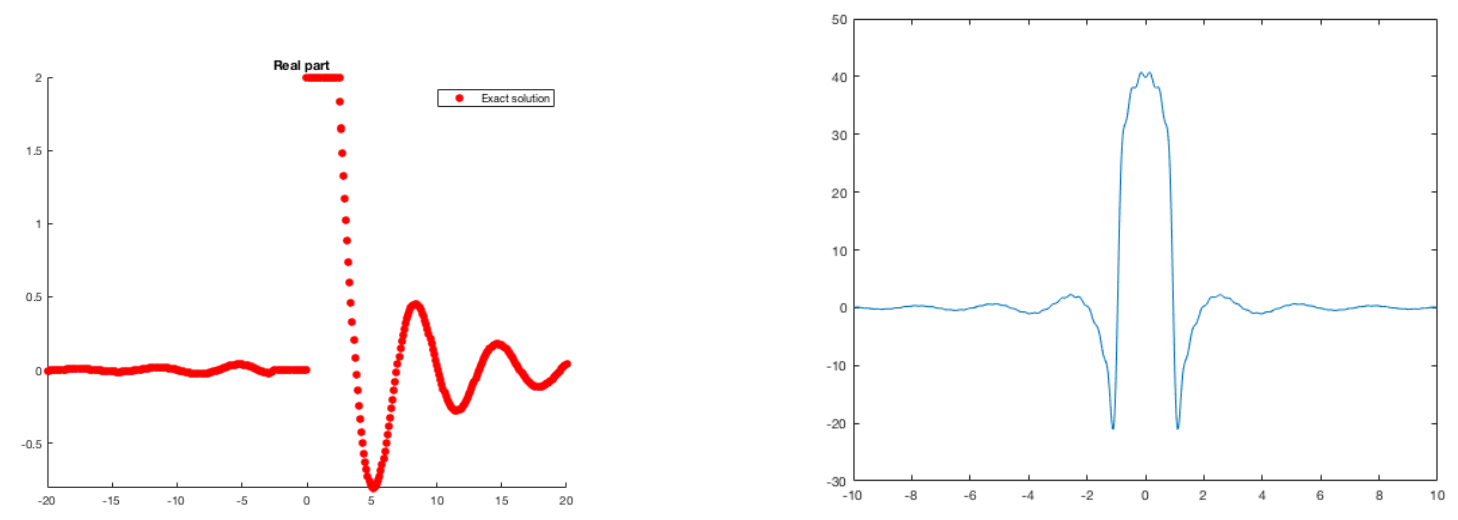

Figure 11: On the left: real part of the computed solution $\varphi_{h}^{0}$ (red points) on $\Sigma^{0}$. On the right: the Fourier transform $\hat{\varphi}^{0}$ for $g$ defined in (78).

The reconstruction in $\Omega$ is shown in Figure 12. The result is good as there is no visible jump on different reconstructions from different $\varphi^{j}$.

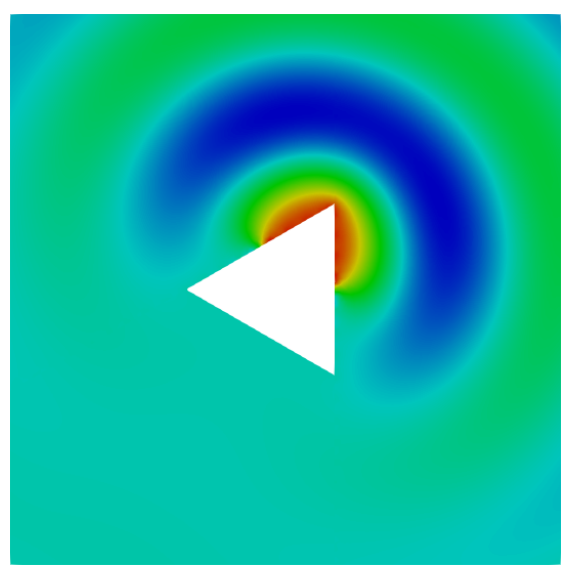

Figure 12: Real part of $p$ with $\alpha=1, \beta=0, \omega=1$, with $g$ given defined in (78).

\subsubsection{Non-dissipative case}

Finally, remark that our theoretical results are established only for $\operatorname{Im}(\omega)>0$. However, the numerical method works for the case without dissipation, provided that we use the representation of the outgoing 
solution in (12) for each half-space, which means that

$$
\sqrt{\omega^{2}-\xi^{2}}= \begin{cases}\sqrt{\left|\omega^{2}-\xi^{2}\right|} & \text { for } \xi^{2}<\omega^{2}, \\ \imath \sqrt{\left|\xi^{2}-\omega^{2}\right|} & \text { for } \xi^{2}>\omega^{2}\end{cases}
$$

To illustrate this, we once again validate the method by using the Hankel function on the boundary of the polygon with $\omega=1$.
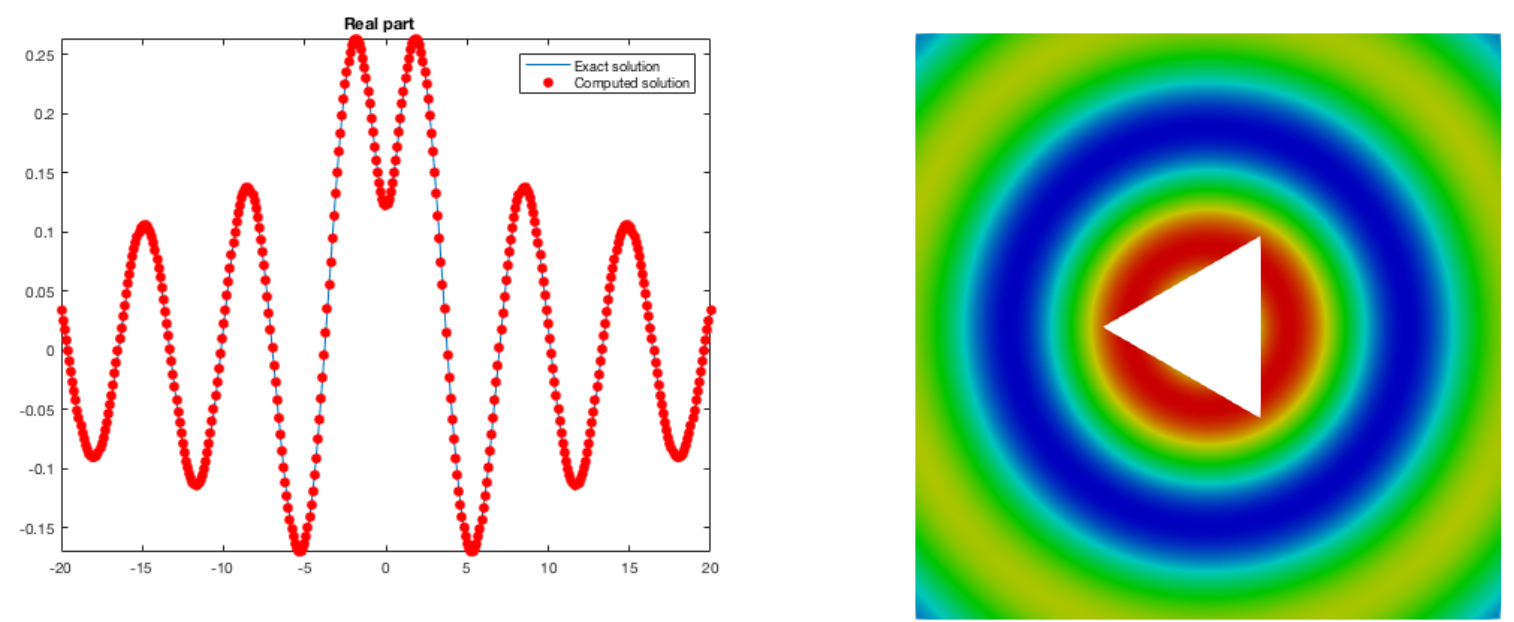

Figure 13: On the left: real part of the computed solution $\varphi_{\mathbf{h}}^{0}$ (red points) on $\Sigma^{0}$. On the right: the reconstruction of the solution with $\omega=1$.

In Figure 13 on the left, as expected, we see that the solution decreases more slowly compared to the case with dissipation. The computed solution matches the exact solution well and the $L^{2}$ relative error on the lines is $1.50 \%$, which is higher than the case with dissipation $(\operatorname{Im} \omega>0)$. The reconstructed solution on the domain has an $L^{2}$ relative error of $0.79 \%$.

We also show here the solution of a scattering problem with an incident wave

$$
p_{i n c}=e^{\imath \omega(x \cos \gamma+y \sin \gamma)}
$$

with $\gamma=3 \pi / 4$. The scattered field is solution of (1) with the boundary data is

$$
g=-\alpha p_{i n c}-\beta \frac{\partial p_{i n c}}{\partial n} \text { on } \partial \mathcal{O}
$$

We consider the solution obtained with the parameters $T=40, h=0.05$, and $\hat{T}=10$ as the "exact solution" (represented on Figure 14 (right)) and we plot the error for different value of $\hat{T}$ between 1 and 8. According to error estimate (74), we expect a behavior like

$$
\operatorname{err} \sim \frac{1}{\hat{T}^{s_{0}}},
$$

where $s_{0}$ is the supremum of $s$ values such that all traces $\varphi^{j}$ belong to $H^{s}\left(\Sigma^{j}\right)$. Here, the theory of singularities [14] shows that $p \in H^{8 / 5}(\Omega)$, so that, taking its normal derivative, we get $s_{0}=1 / 10$. In fact, we observe on Figure 14 (left) that the error decreases more rapidly like

$$
\operatorname{err} \sim \frac{1}{\hat{T}^{2}}
$$

It is probably due to the discretization in space that cannot capture the singularity at the corner.

Acknowledgement: the authors want to thank the anonymous referees for their interest for this work, their relevant remarks, and their suggestions to enhance the readability of the paper. 

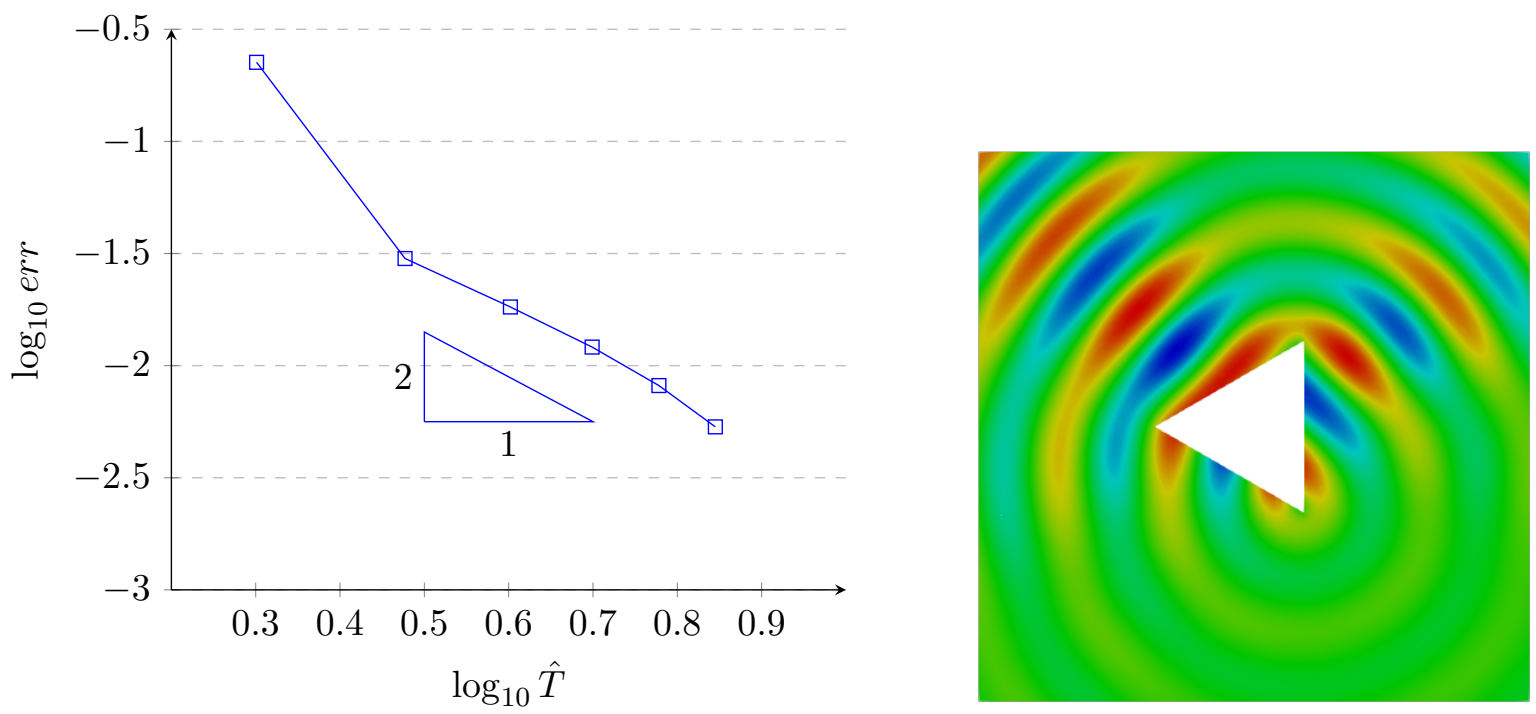

Figure 14: On the left: Influence of the length of the Fourier integral $\hat{T}$ in the scattering problem. On the right: real part of the scattered field with $\hat{T}=10$.

\section{A Proof of Lemma 10}

Let us remind the definition of the operator $L_{N}$. For all $\psi \in L^{2}\left(\Sigma^{0}\right)$, we consider the problem

$$
\mid \begin{aligned}
& -\Delta v=0 \quad \text { in } \Omega^{0}, \\
& \beta \frac{\partial v}{\partial x}=\psi \quad \text { on } \Sigma^{0} .
\end{aligned}
$$

This problem has a unique solution $v$ in the following weighted Sobolev space (see for instance [17, Chap. 6]):

$$
\left\{u \in L_{l o c}^{2}\left(\Omega^{0}\right) \mid \frac{1}{r^{3 / 2}} u \in L^{2}\left(\Omega^{0}\right), \frac{1}{\sqrt{r}} \nabla u \in L^{2}\left(\Omega^{0}\right)\right\} .
$$

The operator $L_{N}$ is defined as

$$
\begin{aligned}
L_{N}: L^{2}\left(\Sigma^{0}\right) & \rightarrow L^{2}\left(\Sigma^{1} \cap \Omega^{0}\right), \\
L_{N} \psi & :=\left.\beta \frac{\partial}{\partial x^{1}} v(\psi)\right|_{\Sigma^{1} \cap \Omega^{0}} .
\end{aligned}
$$

We want to show in this appendix that

1. The operator $L_{N}$ is continuous from $L^{2}\left(\Sigma^{0}\right)$ in $L^{2}\left(\Sigma^{1} \cap \Omega^{0}\right)$ and its norm is bounded by 1 ;

2. $\exists C \in(0,1), \quad \forall \psi \in L^{2}\left(\Sigma^{0}\right), \quad\left\|L_{N} \chi_{\left(a_{0}^{+},+\infty\right)} \psi\right\| \leq C\left\|\chi_{\left(a_{0}^{+},+\infty\right)} \psi\right\|$;

3. $L_{N} \chi_{\left(-\infty, a_{0}^{-}\right)}$is a compact operator from $L^{2}\left(\Sigma^{0}\right)$ in $L^{2}\left(\Sigma^{1} \cap \Omega^{0}\right)$.

Let us begin the proof which is based on Mellin techniques.

1. Denoting $(r, \theta)$ the polar coordinates (the center is the intersection point between $\Sigma^{0}$ and $\left(\Sigma^{1} \cap\right.$ $\left.\Omega^{0}\right)$ ) defined by

$$
\mid \begin{aligned}
& r=\sqrt{\left(x-l^{0}\right)^{2}+\left(y-a_{0}^{+}\right)^{2}} \in(0,+\infty), \\
& \theta=\frac{\pi}{2}-\arctan \left(\frac{y-a_{0}^{+}}{x-l^{0}}\right) \in(0, \pi) .
\end{aligned}
$$


We introduce the function $w$ defined for almost everywhere $(t, \theta) \in \mathcal{B} \equiv \mathbb{R} \times(0, \pi)$ by $w(t, \theta)=$ $v(x, y)$ where $t=\ln r$ and $(r, \theta)$ is defined in (80). It is the solution of

$$
\mid \begin{aligned}
-\Delta_{t, \theta} w=0 & \text { in } \mathcal{B}, \\
\beta \frac{\partial w}{\partial \theta}(t, 0) & =\psi_{0}(t):=e^{t} \psi\left(e^{t}+a_{0}^{+}\right) \\
-\beta \frac{\partial w}{\partial \theta}(t, \pi) & =\psi_{\pi}(t):=e^{t} \psi\left(-e^{t}+a_{0}^{+}\right) .
\end{aligned}
$$

We can show by a simple change of variable that

$$
\psi \in L^{2}\left(\Sigma^{0}\right) \quad \Rightarrow \quad t \mapsto e^{-t / 2} \psi_{0} \in L^{2}(\mathbb{R}) \quad \text { and } \quad t \mapsto e^{-t / 2} \psi_{\pi} \in L^{2}(\mathbb{R})
$$

and

$$
\left\|e^{-t / 2} \psi_{0}\right\|_{L^{2}(\mathbb{R})}=\|\psi\|_{L^{2}\left(a_{0}^{+},+\infty\right)} \quad \text { and } \quad\left\|e^{-t / 2} \psi_{\pi}\right\|_{L^{2}(\mathbb{R})}=\|\psi\|_{L^{2}\left(-\infty, a_{0}^{+}\right)} .
$$

It is possible to compute explicitly $w$ by applying the Fourier-Laplace transform which is defined as

$$
\check{u}(\lambda) \equiv\left[\mathcal{M}_{t \rightarrow \lambda}\right](\lambda):=\int_{\mathbb{R}} e^{-\lambda t} u(t) \mathrm{d} t .
$$

It is an isomorphism between $\left\{u, e^{\gamma t} u \in L^{2}(\mathbb{R})\right\}$ and $L^{2}\left(l_{-\gamma}\right)$ where $l_{-\gamma}=\{\lambda=-\gamma+i s, s \in \mathbb{R}\}$, for all $\gamma \in \mathbb{R}$ and we have the Plancherel formula

$$
\int_{\mathbb{R}} e^{2 \gamma t}|u(t)|^{2} \mathrm{~d} t=\frac{1}{2 \pi i} \int_{l_{-\gamma}}|\check{u}(\lambda)|^{2} \mathrm{~d} \lambda:=\|\check{u}\|_{L^{2}\left(l_{-\gamma}\right)}^{2} .
$$

We have in particular thanks to (82-83)

$$
\begin{array}{ll}
\lambda \mapsto \check{\psi}_{0}(\lambda) \in L^{2}\left(l_{1 / 2}\right), \quad\left\|\check{\psi}_{0}\right\|_{L^{2}\left(l_{1 / 2}\right)}=\|\psi\|_{L^{2}\left(a_{0}^{+},+\infty\right)}, \\
\lambda \mapsto \check{\psi}_{\pi}(\lambda) \in L^{2}\left(l_{1 / 2}\right), \quad\left\|\check{\psi}_{\pi}\right\|_{L^{2}\left(l_{1 / 2}\right)}=\|\psi\|_{L^{2}\left(-\infty, a_{0}^{+}\right)}
\end{array}
$$

Applying the Fourier-Laplace transform to $w$, we have $\check{w}(\bullet, \theta)=\mathcal{M}_{t \mapsto \lambda} w(\bullet, \theta)$ which satistifes

$$
\begin{aligned}
-\lambda^{2} \check{w}(\lambda, \theta)-\frac{\partial^{2} \check{w}}{\partial \theta^{2}}(\lambda, \theta) & =0, \quad \forall \lambda \in \mathbb{C} \\
\beta \frac{\partial \check{w}}{\partial \theta}(\lambda, 0) & =\check{\psi}_{0}(\lambda), \\
-\beta \frac{\partial \check{w}}{\partial \theta}(\lambda, \pi) & =\check{\psi}_{\pi}(\lambda),
\end{aligned}
$$

and we can easily find the solution of this equation. We obtain for $\lambda \notin \mathbb{Z}$

$$
\check{w}(\lambda, \theta)=\frac{\cos (\lambda(\pi-\theta))}{\beta \lambda \sin (\lambda \pi)} \check{\psi}_{0}(\lambda)+\frac{\cos (\lambda \theta)}{\beta \lambda \sin (\lambda \pi)} \check{\psi}_{\pi}(\lambda),
$$

which, for $\lambda \notin \mathbb{Z}$, leads to

$$
\beta \frac{\partial \check{w}}{\partial \theta}(\lambda, \theta)=A(\lambda, \pi-\theta) \check{\psi}_{0}(\lambda)-A(\lambda, \theta) \check{\psi}_{\pi}(\lambda)
$$

where

$$
A(\lambda, \theta)=\frac{\sin (\lambda \theta)}{\sin (\lambda \pi)}
$$

Moreover, we can show that for all $\theta \in(0, \pi), s \mapsto|A(1 / 2+\imath s, \theta)|$ is in $L^{\infty}(\mathbb{R})$ and its supremum is attained at $s=0$ and it is equal to $\sin (\theta / 2)$. Using the Cauchy Schwartz inequality $(a b+c d)^{2} \leq$ $\left(a^{2}+c^{2}\right)\left(b^{2}+d^{2}\right)$, we have then, for all $\theta \in(0, \pi)$

$$
\begin{aligned}
\left\|\beta \frac{\partial \check{w}}{\partial \theta}(\lambda, \theta)\right\|_{L^{2}\left(l_{1 / 2}\right)}^{2} & \leq\left(\cos (\theta / 2)\left\|\check{\psi}_{0}\right\|_{L^{2}\left(l_{1 / 2}\right)}-\sin (\theta / 2)\left\|\check{\psi}_{\pi}\right\|_{L^{2}\left(l_{1 / 2}\right)}\right)^{2} \\
& \leq\left(\left\|\check{\psi}_{0}\right\|_{L^{2}\left(l_{1 / 2}\right)}^{2}+\left\|\check{\psi}_{\pi}\right\|_{L^{2}\left(l_{1 / 2}\right)}^{2}\right)=\|\psi\|_{L^{2}\left(\Sigma^{0}\right)}^{2} .
\end{aligned}
$$


The last inequality for $\theta=\theta^{0,1}$, after the change of variable $r=e^{t}$, yields to

$$
\left.\beta \frac{\partial v}{\partial x^{1}}\right|_{\Sigma^{1} \cap \Omega^{0}} \in L^{2}\left(\Sigma^{1} \cap \Omega^{0}\right)
$$

and

$$
\left\|\left.\beta \frac{\partial v}{\partial x^{1}}\right|_{\Sigma^{1} \cap \Omega^{0}}\right\|_{L^{2}\left(\Sigma^{1} \cap \Omega^{0}\right)} \leq\|\psi\|_{L^{2}\left(\Sigma^{0}\right)} .
$$

We have shown that the operator $L_{N}$ is continuous from $L^{2}\left(\Sigma^{0}\right)$ to $L^{2}\left(\Sigma^{1} \cap \Omega^{0}\right)$ and its norm is bounded by 1 .

2. The norm of $L_{N} \chi_{\left(a^{0},+\infty\right)}$ can be deduced from the previous computation by taking $\psi_{\left(-\infty, a_{0}^{+}\right)}=0$ or equivalently $\check{\psi}_{\pi}=0$. We get for all $\theta \in(0, \pi)$

$$
\left\|\beta \frac{\partial \breve{w}}{\partial \theta}(\lambda, \theta)\right\|_{L^{2}\left(l_{1 / 2}\right)} \leq \cos (\theta / 2)\left\|\check{\psi}_{0}(\lambda)\right\|_{L^{2}\left(l_{1 / 2}\right)}=\cos (\theta / 2)\|\psi\|_{L^{2}\left(a_{0}^{+},+\infty\right)} .
$$

from where we conclude that the norm of the operator $L_{N} \chi_{\left(a^{0},+\infty\right)}$ is bounded by $\cos \left(\theta^{0,1} / 2\right)$.

3. Finally let us consider the previous computation with $\psi=0$ on $\left(a_{0}^{-},+\infty\right)$. This corresponds to take $\psi_{0}=0$ and $\psi_{\pi}=e^{t} \psi\left(-e^{t}+a_{0}^{+}\right)$. Since $\psi$ vanishes on $\left(a_{0}^{-},+\infty\right)$, we have $e^{-\gamma t} \psi_{\pi}$ is in $L^{2}(\mathbb{R})$ for any $\gamma>1$ and so, by $(85), \breve{\psi}_{\pi}$ is in $L^{2}\left(l_{\gamma}\right)$ for all $\gamma>1$. The previous computation yields to

$$
\forall \lambda \notin \mathbb{Z}, \quad \beta \frac{\partial \check{w}}{\partial \theta}\left(\lambda, \theta^{0,1}\right)=-A\left(\lambda, \theta^{0,1}\right) \check{\psi}_{\pi}(\lambda)
$$

We can show that

$$
\forall \gamma>1, \gamma \notin \mathbb{N}, \quad \sup _{\lambda \in l_{\gamma}} \lambda A\left(\lambda, \theta^{0,1}\right)<+\infty,
$$

which enables us to deduce that

$$
\forall \gamma>1, \gamma \notin \mathbb{N}, \quad \lambda \frac{\partial \check{w}}{\partial \theta}\left(\lambda, \theta^{0,1}\right) \in L^{2}\left(l_{\gamma}\right) .
$$

By applying inverse Laplace-Fourier transform, we have then

$$
\forall \gamma>1, \gamma \notin \mathbb{N}, \quad e^{-\gamma t} \frac{\partial^{2} \check{w}}{\partial t \partial \theta}\left(t, \theta^{0,1}\right) \in L^{2}(\mathbb{R}),
$$

and by change of variable,

$$
\forall \gamma>1, \gamma \notin \mathbb{N}, \quad r^{-\gamma+3 / 2} \partial_{r}\left(\frac{1}{r} \frac{\partial \check{w}}{\partial \theta}\left(r, \theta^{0,1}\right)\right) \in L^{2}\left(\mathbb{R}^{+}\right) .
$$

If we choose $\gamma=3 / 2$, the operator $L_{N} \chi_{\left(-\infty, a_{0}^{-}\right)} \in H^{1}(0,+\infty)$. By compact embedding of $H^{1}(0, b)$ in $L^{2}(0, b)$ for any $b>0$, we show that $\chi_{(0, b)} L_{N} \chi_{\left(-\infty, a_{0}^{-}\right)}$is compact. It suffices to use similar argument as in the proof of Lemma 8 to show that $\chi_{(b,+\infty)} L_{N} \chi_{\left(-\infty, a_{0}^{-}\right)}$is a Hilbert-Schmidt operator.

Let us now give other properties of $L_{N}$ which will be useful for the numerical analysis (see Section 4). We remind the definition of the symmetric and anti-symmetric operators, defined in the proof of Lemma 17, $S$ and $A \in \mathcal{L}\left(L^{2}(\mathbb{R})\right)$

$$
\forall \psi \in L^{2}(\mathbb{R}), \quad S \psi(y)=\frac{1}{2}\left(\psi(y)+\psi\left(2 a_{0}^{+}-y\right)\right), \quad \text { and } A \psi(y)=\frac{1}{2}\left(\psi(y)-\psi\left(2 a_{0}^{+}-y\right)\right)
$$

We remind that $S+A=I d$. , $S^{2}=S, A^{2}=A,\|S\| \leq 1,\|A\| \leq 1$ and for any $\psi \in L^{2}(\mathbb{R})$ such that $\psi_{\left(-\infty, a_{0}^{+}\right)}=0$, we have

$$
\|S \psi\|_{L^{2}(\mathbb{R})}=\|A \psi\|_{L^{2}(\mathbb{R})}=\frac{1}{\sqrt{2}}\|\psi\|_{L^{2}(\mathbb{R})}
$$


Let us now study the norm of $L_{N} S \psi$ and $L_{N} A \psi$ for any $\psi \in L^{2}(\mathbb{R})$. By reproducing the previous calculations, we have easily that, writing $\overline{(S \psi)_{0}}=\overline{(S \psi)_{\pi}}$,

$$
\left\|L_{N} S \psi\right\|_{L^{2}\left(\Sigma^{1} \cap \Omega^{0}\right)} \leq \sup _{\lambda \in l_{1 / 2}}\left|A\left(\lambda, \pi-\theta^{0,1}\right)-A\left(\lambda, \theta^{0,1}\right)\right|\|S \psi\|_{L^{2}(a,+\infty)}
$$

where we remind that

$$
\forall \theta \in(0, \pi), \forall \lambda \notin \mathbb{Z}, \quad A(\lambda, \theta)=\frac{\sin (\lambda \theta)}{\sin (\lambda \pi)} .
$$

We can show that the supremum is attained at $\lambda=1 / 2$ and then

$$
\sup _{\lambda \in l_{1 / 2}}\left|A\left(\lambda, \pi-\theta^{0,1}\right)-A\left(\lambda, \theta^{0,1}\right)\right|=\left|\cos \left(\theta^{0,1} / 2\right)-\sin \left(\theta^{0,1} / 2\right)\right| .
$$

Using that $\sqrt{2}\|S \psi\|_{L^{2}(a,+\infty)}=\|S \psi\|_{L^{2}(\mathbb{R})} \leq\|\psi\|_{L^{2}(\mathbb{R})}$, we obtain

$$
\left\|L_{N} S \psi\right\|_{L^{2}\left(\Sigma^{1} \cap \Omega^{0}\right)} \leq \frac{\left|\cos \left(\theta^{0,1} / 2\right)-\sin \left(\theta^{0,1} / 2\right)\right|}{\sqrt{2}}\|\psi\|_{L^{2}(\mathbb{R})} .
$$

Similarly, we get

$$
\left\|L_{N} A \psi\right\|_{L^{2}\left(\Sigma^{1} \cap \Omega^{0}\right)} \leq \frac{\cos \left(\theta^{0,1} / 2\right)+\sin \left(\theta^{0,1} / 2\right)}{\sqrt{2}}\|\psi\|_{L^{2}(\mathbb{R})} .
$$

Moreover, let us remark, that gathering these inequalities, we obtain an inequality which comparing to $(87)$, is not optimal :

$\left\|L_{N} \psi\right\|_{L^{2}\left(\Sigma^{1} \cap \Omega^{0}\right)} \leq\left\|L_{N} S \psi\right\|_{L^{2}\left(\Sigma^{1} \cap \Omega^{0}\right)}+\left\|L_{N} A \psi\right\|_{L^{2}\left(\Sigma^{1} \cap \Omega^{0}\right)} \leq \sqrt{2} \max \left(\cos \left(\theta^{0,1} / 2\right), \sin \left(\theta^{0,1} / 2\right)\right)\|\psi\|_{L^{2}(\mathbb{R})}$.

Moreover, for $\psi \in L^{2}(\mathbb{R})$ such that $\psi_{\left(-\infty, a_{0}^{+}\right)}=0$, using (89), we obtain

$$
\left\|L_{N} \psi\right\|_{L^{2}\left(\Sigma^{1} \cap \Omega^{0}\right)} \leq\left\|L_{N} S\right\|\|S \psi\|_{L^{2}(\mathbb{R})}+\left\|L_{N} A\right\|\|A \psi\|_{L^{2}(\mathbb{R})} \leq C^{\prime}\|\psi\|_{L^{2}(\mathbb{R})}
$$

where

$$
C^{\prime}=\max \left(\cos \left(\theta^{0,1} / 2\right), \sin \left(\theta^{0,1} / 2\right)\right) \in(0,1) .
$$

This result is then not optimal for $\theta^{0,1} \in(0, \pi / 2)$ (compared to (88)) but the constant $C^{\prime}$, obtained that way, is still in $(0,1)$.

\section{References}

[1] Apel T., Nicaise S., and Pfefferer J. (2015), Discretization of the Poisson equation with nonsmooth data and emphasis on non convex domains, arXiv.

[2] Adams R.A. and Fournier J.J.F. (2003), Sobolev Spaces, Elsevier Science.

[3] Amos D.E. (1985), A Subroutine Package for Bessel Functions of a Complex Argument and Nonnegative Order, Sandia National Laboratory Report, SAND85-1018.

[4] Atkinson K. E. (1997), The Numerical Solution of Integral Equations of The Second Kind, Cambridge University Press.

[5] Bécache E., Fauqueux S., and Joly P. (2003), Stability of perfectly matched layers, group velocities and anisotropic waves, Journal of Computational Physics, DOI: 10.1016/S0021-9991(03)00184-0, vol. 188(2), pp 399-433.

[6] Besse C., Coatléven J., Fliss S., Lacroix-Violet I., and Ramdani K. (2013), Transparent boundary conditions for locally perturbed infinite hexagonal periodic media, Communications in Mathematical Sciences, vol. 11 (4), pp 907-938. 
[7] Bonnet-Ben Dhia A.-S., Chambeyron C., and Legendre G. (2014), On the use of perfectly matched layers in the presence of long or backward guided elastic waves, Wave Motion, DOI: 10.1016/j.wavemoti.2013.08.001, vol. 51 (2), pp 266-283.

[8] Bonnet-Ben Dhia A.-S., Fliss S., and Tonnoir A. (2018), The halfspace matching method: a new method to solve scattering problem in infinite media, JCAM vol. 338, pp. 44-68.

[9] Chandler G. A. (1984), Galerkin's method for boundary integral equations on polygonal domains, Journal of The Australian Mathematical Society, Series B, 26. pp. 1-13.

[10] Chandler-Wilde S. N., Hothersall D. C. (1995), Efficient calculation of the green function for acoustic propagation above a homogeneous impedance plane, Journal of Sound and Vibration, vol. 180 (5), pp. 705-724.

[11] Chandler-Wilde S. N., Langdon S., and Mokgolele M. (2012), A high frequency boundary element method for scattering by convex polygons with impedance boundary conditions, Communications in Computational Physics, vol. 11 (2), pp. 573-593. ISSN 1991-7120.

[12] Dauge M. (2006), Elliptic Boundary Value Problems on Corner Domains: Smoothness and Asymptotics of Solutions, Springer Berlin Heidelberg.

[13] Fliss S. and Joly P. (2009), Exact boundary conditions for time-harmonic wave propagation in locally perturbed periodic media, Applied Numerical Mathematics, vol. 59 (9), pp. 2155-2178.

[14] Grisvard P. (1992), Singularities in boundary value problems, Springer Berlin Heidelberg.

[15] Grisvard P. (2011), Elliptic problems in nonsmooth domains, Society for Industrial and Applied Mathematics vol. 69 .

[16] Kielbasiewicz N. and Lunéville E. (2016), User documentation of XLiFE++, POEMS, ENSTA.

[17] Kozlov V. A., Mazia V. G., and Rossmann J. (1997), Elliptic boundary value problems in domains with point singularities, American Mathematical Soc., vol. 52.

[18] Lenoir M. and Tounsi A. (1988), The localized finite element method and its application to the two-dimensional sea-keeping problem, SIAM J. NUMER. ANAL. vol. 25 (4).

[19] Reed M. and Simon B. (1980), I: Functional Analysis, Elsevier, vol. 1.

[20] Tonnoir A. (2015), Transparent conditions for the diffraction of elastic waves in anisotropic media, Ecole Polytechnique, PhD Thesis.

[21] Velichko A. and Wilcox P. D. (2007), Modeling the excitation of guided waves in generally anisotropic multilayered media, The Journal of the Acoustical Society of America, vol. 121(1), pp. 60-69. 